\title{
Effects of blade rotation direction in the wake region of two in-line turbines using Large Eddy Simulation
}

\author{
Amin Allah Veisi \\ PhD student, Department of Mechanical Engineering, University of Sistan and Baluchestan, Zahedan, Iran. \\ Amin_veysi@yahoo.com \\ Mohammad Hossein Shafiei Mayam* \\ Assistant Professor, Department of Mechanical Engineering, Bozorgmehr University of Qaenat, Iran \\ shafiei@buqaen.ac.ir
}

\begin{abstract}
In the present work, flow around a horizontal axis wind turbine is investigated in co-rotating and counter-rotating configurations. Large Eddy Simulation (LES) has been employed in order to study the effect of rotation direction of the downstream turbine in a two in-line setup. The results are in good agreement with presenting experimental data in literatures. The power efficiency of the downstream turbine has increased about $4 \%$ in the counterrotating configuration rather than co-rotating configuration with the same separation distance (3D). The power efficiency of the downstream turbine increased about $7 \%$ by decreasing the separation distance to 1D. The improved efficiency in the counter-rotating configuration is due to the flow rotation direction of the upstream turbine. The results revealed in both configurations that streamwise velocity is almost identical, while lateral velocities are changed greatly. Lateral velocities are decreased by moving in a downstream direction. Hence, the benefit of this configuration is obtained by lower separation distance. The authors suggested counter-rotating configuration for the terrain with a high density of installed wind turbines and believed that operating the wind turbines in counter-rotating configuration will not only improve the total efficiency of two in-line turbines, but will also considerably reduce the space they require. Since the oscillations of power coefficient are so high, the exact location of encountering the vortices on downstream blades should be determined to improve the power coefficient of two in line turbines. So, further studies are suggested to extract and track the vortices for future work to increase the efficiency of wind farms.
\end{abstract}




\section{Keywords:}

Large Eddy Simulation, Wind turbine, Wind energy, Turbulence Structures

\section{Introduction}

Fossil fuels, which are declining, are the largest sources of carbon dioxide emissions and the main cause of global warming. Renewable energies are an alternative for fossil resources as they can alleviate heat-trapping in the atmosphere. Among the renewable energies, wind energy is a cheaper alternative and is the fastest energy growing energy resource $[1,2]$. This type of energy causes no environmental pollution and is widely distributed all over the world [3]. Thus, the field of wind power is attractive to many researchers as an energy supplement [4-9]. Wind turbines are separated into two types: horizontal axis and vertical axis [10]. The wake region in the downstream direction of a wind turbine is divided into two different regions: near wake and far wake [11]. The near wake is the region just behind the rotor, and is approximately one rotor diameter downstream of a wind turbine. Here the effects of blade geometry and tip vortices are prevalent. The far wake region is beyond the near wake, where the effects of wind turbine geometry are less important.

The wake interferences between the wind turbines have a significant effect on the performance of wind farms. The downstream wind turbines, in the near wake or far wake regions, are affected by the wake of an upstream wind turbine. Thus, the study of wake development behind the wind turbine blades is important to minimise the effects of wake interaction [12]. The power efficiency of wind farms could be increased by changing the wind turbine arrangement; this issue has been investigated by many scholars [13-20]. For instance, Kuo et al. [18] examined the wind farm layout on complex terrains and proposed a new algorithm for this situation. Park and Law [13] proposed a mathematical optimisation scheme to increase wind farm efficiency. Some studies focus on the separation distances of upstream and downstream turbines. Based on most of them it is shown that better power production will be obtained by increasing the separation distances [14, 21, 22]. Adjusting the pitch angle and yaw angle is also suggested by many researchers [23, 24]. Moreover, detailed knowledge of the flow field and turbulence structures are needed to improve the power production and optimisation of wind farms [11, 12, $19,21,25,26]$. Numerous experimental and numerical studies have been conducted to better understand the flow field. For instance, Van Dooren et al. [27] showed that a short-range Wind Scanner Lidar precisely evaluated small-scale flow structures in a wind tunnel. Zhong et al. [28] found the helical vortices are responsible for higher velocity deficit near the blade tip. The study of the wake region showed that the distinction between the near and far wake regions is influenced by wind speed changes [29]. Also gradual increase of turbulence intensity in the far wake is due to wake instability [30]. The importance of spatial and temporal information of the flow field is shown by many researchers to increase the energy output and lifetime of wind turbines [31-33]. Moreover, many researchers are working on the IEA Task 31 Wakebench platform, which guides the wind energy model developers for evaluation of models [34, 35]. For example Rodrigo et al. [34] evaluated the wind farm flow model over the isolated hill and complex terrain. The results also allow for extending the microscale to mesoscale models in order to complete the atmospheric model evaluation. A comprehensive study on IEA TASK 31 indicated that more detailed knowledge is needed than averaged long time data to better inform differences in model accuracy [35]. 
The goal of the present work is to analysis the interaction between two in-line wind turbines in order to produce more energy when the same conditions and facilities are used. In other words, supplementation of energy is one of the concerns of today's society is on the agenda by increasing the efficiency of the wind farm. So, the authors tried to combine wind turbines at different conditions to achieve the target. As aforementioned, extensive studies have been conducted in order to better understand wake region, use optimised separation distance between turbines and improve the layout of wind farms. In a co-rotating configuration, the efficiency of a wind farm is increased by increasing the separation distance of wind turbines. This arrangement needs more space, while in many countries there are area limitations. So, it is important to resolve this problem. This means the researchers try to increase the efficiency, while the separation distances of wind turbines are not increased. One solution is choosing a counter-rotating configuration. Due to the conservation of angular momentum, flow is rotated in the opposite direction of the upstream turbine. Therefore, the downstream turbine may harvest more energy if it rotates in the opposite direction of the upstream turbine. To study this phenomenon, the main goal of the present work focuses on the effect of the rotation direction of downstream turbines in a counter-rotating configuration. For this reason, the LES method is employed to study the flow. First, it is necessary to validate the results of LES using reliable data in the literature.

Based on the above-mentioned processes, the present work is based on the "blind test" workshop results [36, 37]. First, LES has been performed around a single wind turbine that rotates in a counter clockwise direction when observed from the upstream side at different rotational speed. A single wind turbine is also tested that is rotated in a clockwise direction. The blade of this turbine is made by mirroring the counter-clockwise blade in order to rotate in a clockwise direction. Then, two in-line wind turbines are tested with two different co-rotating and counter-rotating configurations (Fig.1). Moreover, the effect of wind turbine distances is studied. Wake development, velocity changes, turbulence intensity and swirling strength are studied behind the wind turbine in order to attain detailed information on fluid flow in the wake region of both configurations.

\section{Numerical simulation}

\subsection{Wind tunnel and model of wind turbines}

The wake development is studied in terms of how two similar turbines operate in-line in the "blind test 2" workshop (BT2) [37]. Both upstream and downstream wind turbines are similar and have the same geometry in co-rotating and counter-rotating configurations, but slightly different hub sizes, which leads to somewhat different rotor diameters of $D_{1}=0.944 m$ while $D_{2}=0.894 m$. NREL $S 826$ airfoil is used to build the blade along the entire span of the blade. This profile has a high lift coefficient and low sensitivity to roughness [38].

The airfoil section is shown in Fig 2a. The NREL Sde826 wind turbine blade is shown in Fig. 2b (see [39] for further details). The used wind tunnel in this study has a cross section of $\mathrm{L}_{X}=2.7 \mathrm{~m}$, a height of $\mathrm{L}_{Y}=1.8 \mathrm{~m}$ and the total length is $\mathrm{L}_{Z}=11.14 \mathrm{~m}$. The wind turbine is located $2 \mathrm{D}$ rotor diameters $\left(\mathrm{L}_{\mathrm{Z} 1}\right)$ after the inlet section of wind tunnels in all conditions. In both configuration turbines have been centred in the wind tunnel. The separation distance is 3D for co-rotating configuration, while the separation distance varied from 3D to 1D for counterrotating configuration. The hub height of the wind turbines from the floor of the wind tunnel is $0.817 \mathrm{~mm}$ in both configurations. 


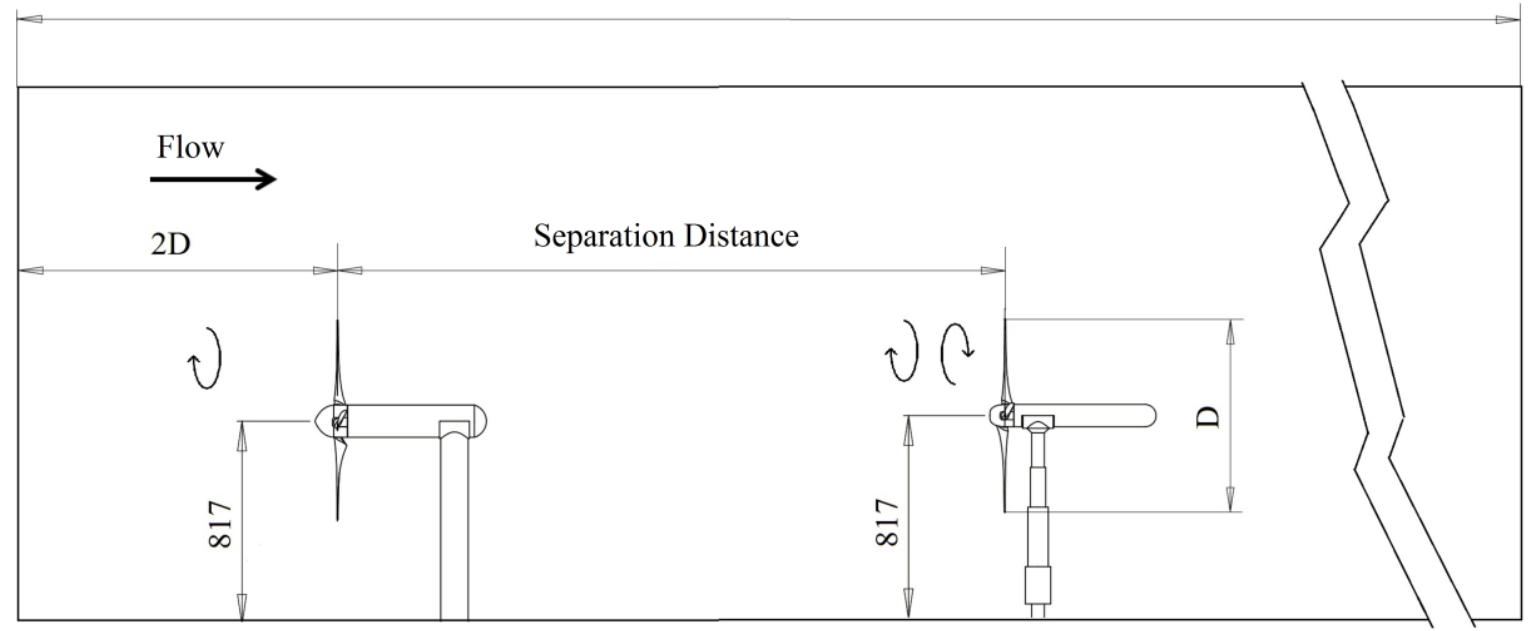

Fig. 1: Co-rotating configuration, the turbines have the same rotation direction, with separation distance of 3D, and in counter-rotating configuration the turbines have opposite rotation direction, the separation distance is varied between $3 \mathrm{D}$ to $1 D$.

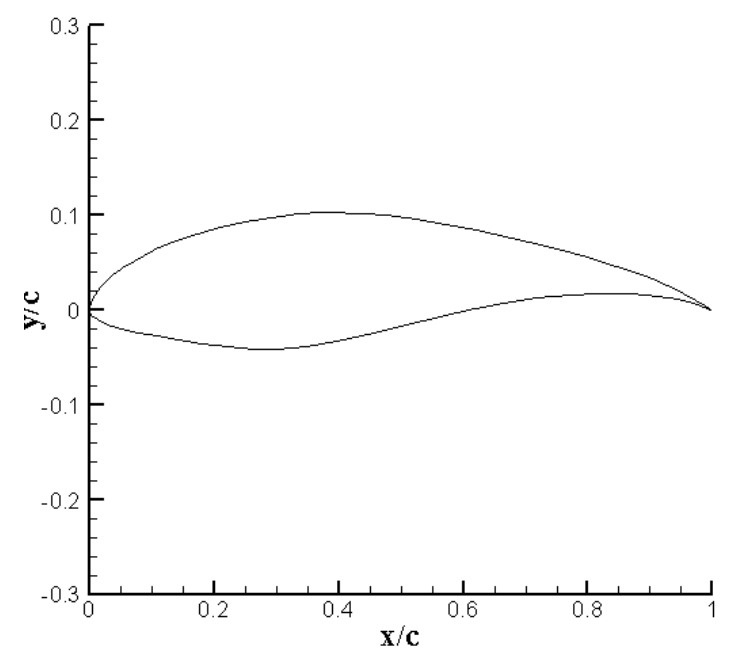

(a)
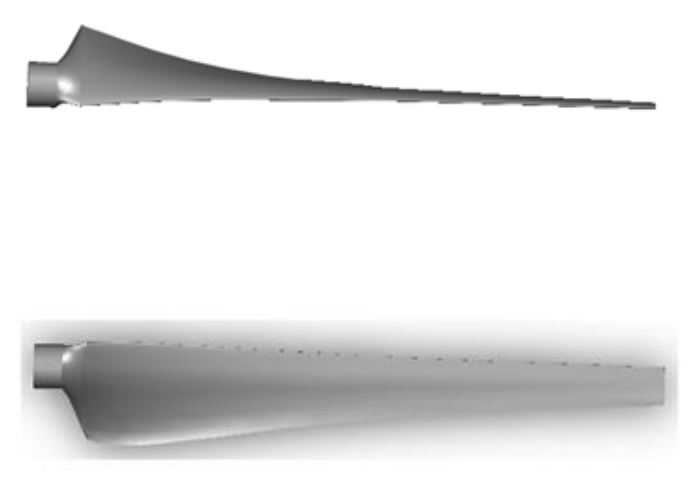

(b)

Fig. 2 (a): NREL S628 airfoil (b) blade in plane of rotation (top) and in the axial direction (down) [39].

\subsection{Boundary conditions and computational mesh}

The inlet condition is considered as a mass driven flow, which is defined with inlet velocity $\left(U_{\text {ref }}=10 \mathrm{~m} / \mathrm{s}\right)$

It should be considered that boundary conditions such as velocity inlet and trivial turbulence intensity at the inlet correspond to the experimental study by the "blind test 2" workshop. For this reason, the turbulence intensity of the flow that enters to the computational domain is $0.3 \%$. Turbulence intensity defined as the ratio of standard deviation to the mean value of the wind speed:

$T I=\frac{\left\langle u^{2}\right\rangle^{1 / 2}}{U_{r e f}}$ 
At the outlet, the ambient pressure condition (zero pressure) is applied. No slip condition is considered at the blades, towers and walls of the wind tunnel. In both setups tip speed ratio of upstream turbine is $\lambda_{1}=6$ and downstream turbine is $\lambda_{2}=4$. Since the angular velocity is not the same in the turbines, the blades of the turbines do not have the same angular position during a revolution. The downstream turbines operate at optimal condition for this rotational speed (see [37] page 6). Tip speed ratio is defined as:

$$
\lambda=\Omega R / U_{r e f}
$$

Where $\Omega$ is angular velocity, $R$ is rotor radius and $U_{r e f}$ is the velocity reference. The test section of the BT2 workshop is simulated in this study. In other words, the computational domain has the same dimension of the mentioned test sections. The domain consists of two moving and stationary zones (Fig 3). The moving zone consists of a cylinder part and wind turbine blades that are located within the stationary zone, which includes the rest of the wind tunnel. The two-cylinder part is considered for the two in-line turbines. The sliding mesh technique is used for the moving zone, unlike the multi-reference frame (MRF) model, which consists of a onelayer interface zone. Finite volume method is taken to discretise the governing equations. The convective terms are discretised using a Bounded Central Differencing scheme. The pressure term is computed using the SIMPLE algorithm. The total number of cells in the numerical grid was $4.6 \times 10^{6}$ in both configurations. The grid refinement factor of 1.1 has been done in the normal direction. The $\mathrm{y}^{+}$value was found to be approximately less than 5 at the blade surface. The time step value of $9.69 \times 10^{-4} \mathrm{sec}$ is considered. This time step is approximately equal to 7 degrees of blade rotation. The number of iterations is equal to 200 and convergence criteria are set to $1 \times 10^{-5}$ for all residuals.

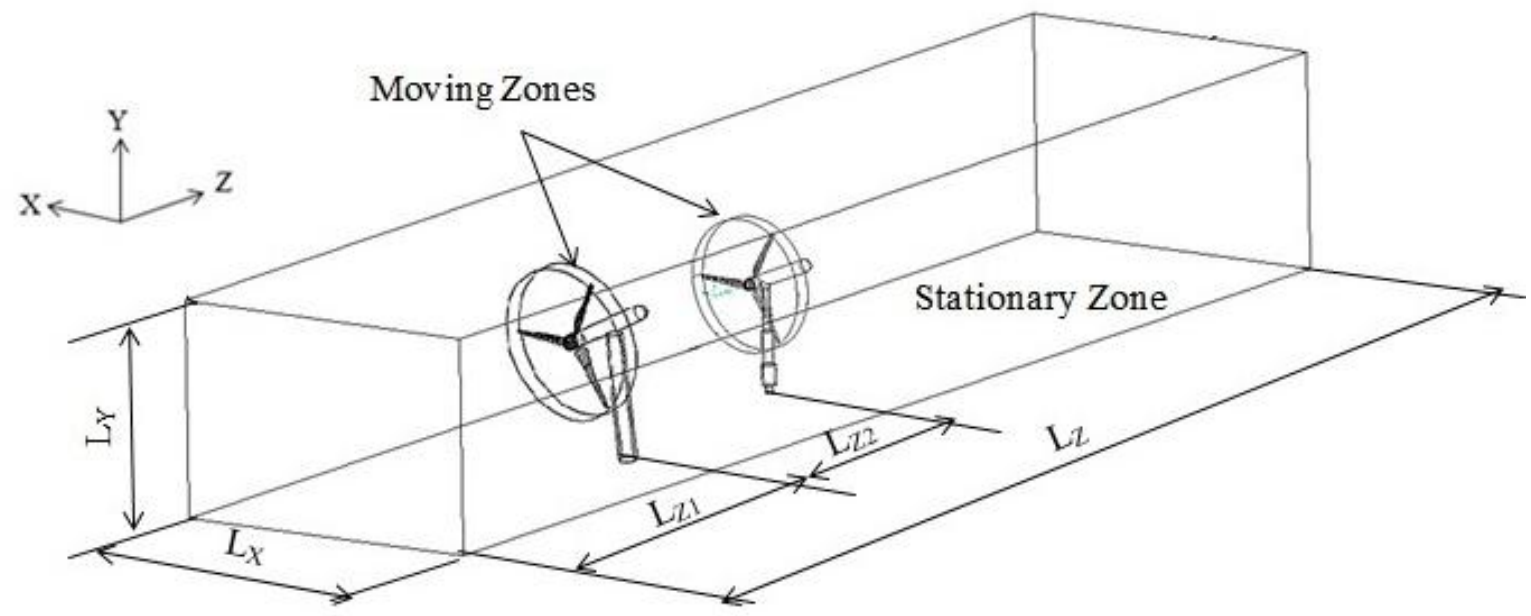

Fig. 3: Wind tunnel placement of the turbines.

\subsection{Governing equations}

Turbulent flow fields are three-dimensional, time-dependent and random. Closure problem is the major issue in turbulent flows, which occur over a wide range of length and time scales. In simulation methods, scales of motion are resolved based on length scales down to grid size in the LES method and to the Kolmogorov scale for Direct Numerical Simulation (DNS). In Reynolds Averaged Navier-Stokes (RANS) all turbulence effects are 
time-averaged and must somehow be modelled. Large scale of motions is directly resolved while small-scale motions are modelled. The LES computational cost lies between Reynolds-Stress Model (RSM) and DNS. RSM is limited to time averaged results. DNS is the most accurate and reliable method for turbulent flows. The DNS method is inapplicable to a large number of engineering problems because DNS cost increases by either the cube of Reynolds number or the geometric complexity. The cause of motivation of the LES method is the limitation of these two approaches. In LES over $80 \%$ of turbulent kinetic energy of the large scale of motion is resolved [40].

The filtered continuum and incompressible Navier-Stokes equations are written as follows:

$$
\begin{aligned}
& \frac{\partial \bar{U}_{i}}{\partial x_{i}}=0 \\
& \frac{\partial \bar{U}_{j}}{\partial t}+\frac{\partial \bar{U}_{i} \bar{U}_{j}}{\partial x_{i}}=v \frac{\partial^{2} \bar{U}_{j}}{\partial x_{i} \partial x_{i}}-\frac{1}{\rho} \frac{\partial \bar{P}}{\partial x_{j}}-\frac{\partial \tau_{i j}^{r}}{\partial x_{i}}
\end{aligned}
$$

where $\bar{U}_{i}$ is the filtered velocity, $\bar{p}$ is the modified filtered pressure field:

$$
\bar{p}=\bar{p}+\frac{2}{3} \rho k_{r}
$$

The residual-stress tensor is defined by the following equations:

$$
\begin{aligned}
\tau_{i j}^{R} & =\overline{U_{i} U_{j}}-\bar{U}_{i} \bar{U}_{j} \\
\tau_{i j}^{r} & =\tau_{i j}^{R}-\frac{2}{3} k_{r} \delta_{i j}
\end{aligned}
$$

Where $\tau_{i j}^{r}$ is the Non- isotropic residual stress tensor and $k_{r}$ is the residual kinetic energy.

\subsection{Residual-stress model}

To overcome the closure problem in LES method a model is needed for the anisotropic residual stress tensor $\tau_{i j}^{r}$. The Smagorinsky model is the simplest model [41]. The residual stress tensor is related to the filtered rate of strain by linear eddy-viscosity:

$$
\begin{aligned}
\tau_{i j}^{r} & =-2 v_{r} \bar{S}_{i j} \\
\bar{S}_{i j} & =\frac{1}{2}\left(\frac{\partial \bar{U}_{i}}{\partial x_{j}}+\frac{\partial \bar{U}_{j}}{\partial x_{i}}\right)
\end{aligned}
$$

Here $v_{r}(\mathrm{x}, \mathrm{t})$ is the eddy viscosity of the residual scales and $\bar{S}_{i j}$ is the filtered rate of strain. The eddy viscosity is modelled in an analogy to the mixing-length hypothesis as below:

$$
\begin{aligned}
v_{r} & =\ell_{s}^{2} \bar{S} \\
& =\left(C_{S} \Delta\right)^{2} \bar{S}
\end{aligned}
$$

Here $\bar{S}$ is the characteristic filtered rate of strain and $\ell_{s}$ is the Smagorinsky length scale. $\Delta$ is the filter width which related to the grid size:

$(\Delta x . \Delta y . \Delta z)^{1 / 3}$ 
Where $\Delta x, \Delta y$ and $\Delta z$ are the mesh size in all directions. In the current study Smagorinsky model with constant coefficient, $C_{S}=0.1$, are used in all conditions.

\section{Results and discussion}

\subsection{Validation}

In a single wind turbine four types of grids were examined: 1.8, 2.7, 3.4 and 4.2 million cells (Fig.4). The results are consistent between mesh 3.4 and 4.2. Since the differences between these two meshes are trivial, the mesh 3.4 is used for further studies. As the wind turbines in the Blind test 2 workshop are similar to the Blind test 1, the same mesh generation procedure was chosen for both tests. Time step or Courant number (which is less than 0.5 ) should be small enough in order to acquire results to track the dynamics of flow and turbulent structures.

In this section, the acquired results of LES in a single turbine and two in-line turbines (co-rotating configuration) are compared with experimental data and two other different numerical simulations for validating the results. Power and thrust coefficients are compared with experimental data at different rotational speed for a single turbine and two in-line turbines (Fig.5 and Fig.6). In a turbulent flow, the velocity field is random, but the Navier-Stokes equations are deterministic. The question is whether the solution is random or deterministic? Turbulent flow is influenced by perturbations of the device, boundary conditions and material properties and shows severe sensitivity to these perturbations. In other words, the device vibrations and surrounding fluctuations are not considered and the boundary conditions of a turbulent flow are incomplete. Hence the solution of the turbulent flow is random. In experimental studies, the perturbation of the turbulent flow can cause separation on the suction side, which reduces the lift force. A part of the fluctuation is modelled in the LES and URANS. In fact, the study of all of the fluctuations is impossible in the flow field. Hence, the possibility of separation on the suction side, lift reduction and incomplete boundary condition could be the reason for the underestimation of the $C_{P}$ coefficient. Moreover, the underestimation of the $C_{P}$ coefficient in other studies can attest to this idea [36].

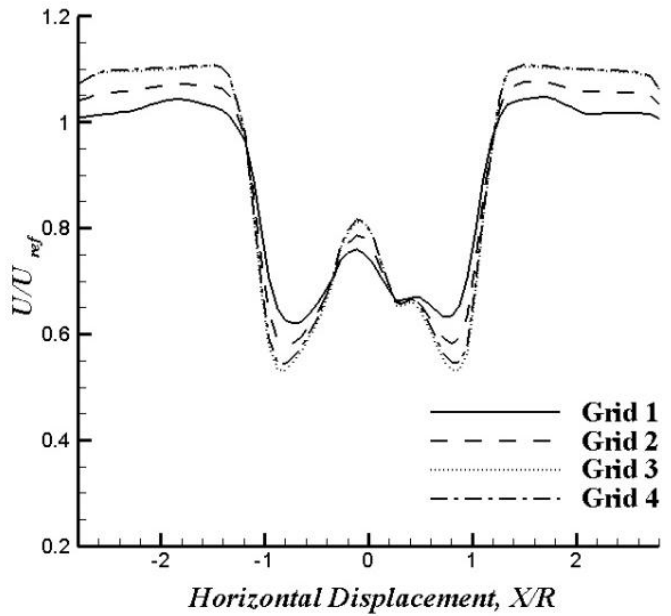

(a)

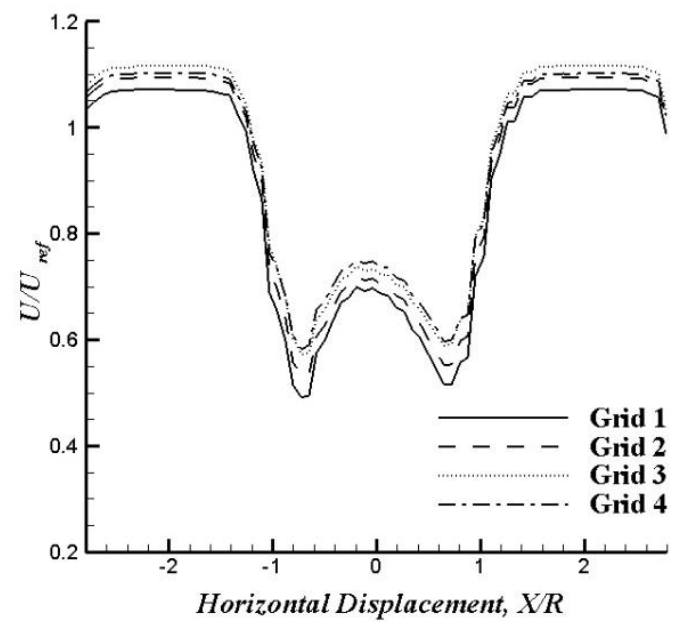

(b)

Fig. 4: Time averaged velocity profile of 4 grids along the horizontal lines centred at 1D (a) and 4D (b) in downstream of a single wind turbine 


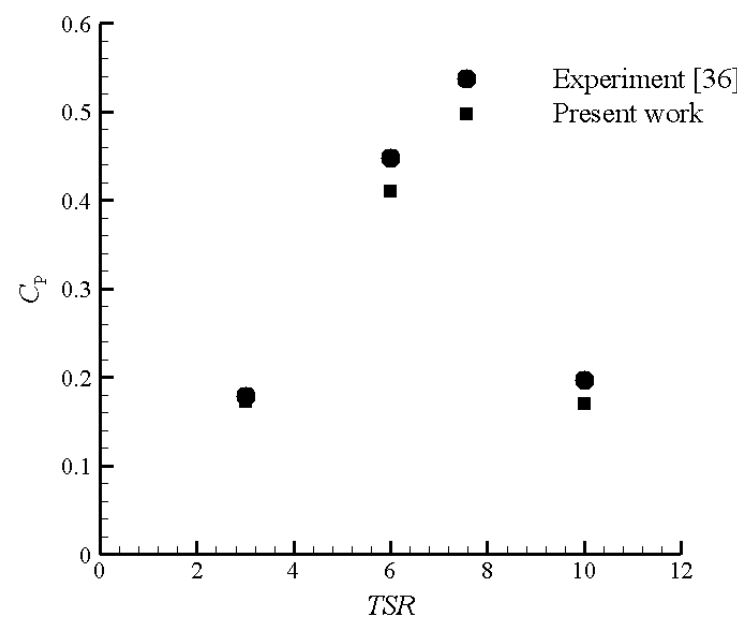

(a)

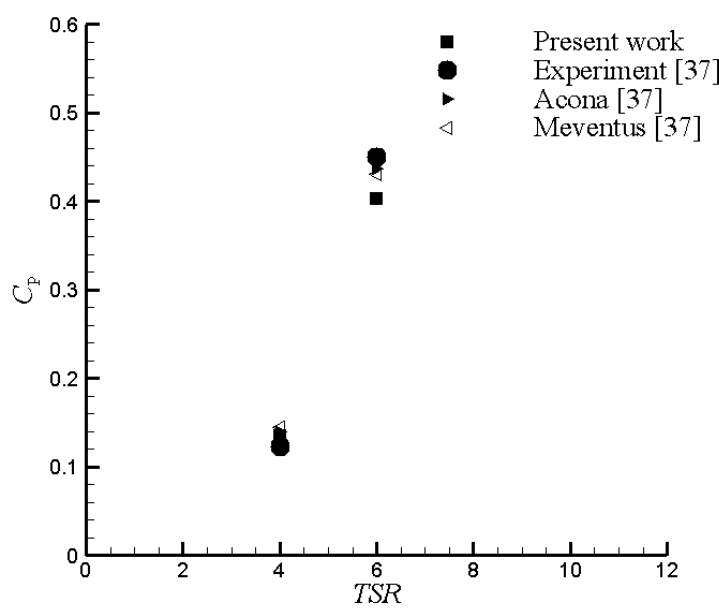

(b)

Fig. 5: $\mathrm{C}_{\mathrm{p}}$ comparison (a) single turbine, (b) two in-line turbines

Time averaged velocity profile along the horizontal lines centred are compared at 1D and 4D in the downstream of two in-line turbines with experimental data and two other numerical simulation results (Fig.7a, b). The results of the present work are in good agreement with the experimental data. The average deviation of the present data from the experimental data is about 2 percent, except at some points whereby the maximum error is around 6 percent.

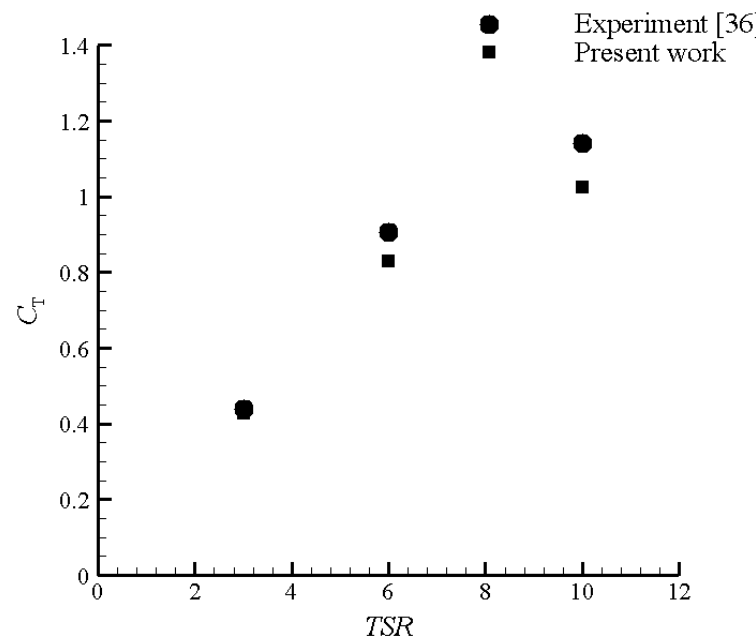

(a)

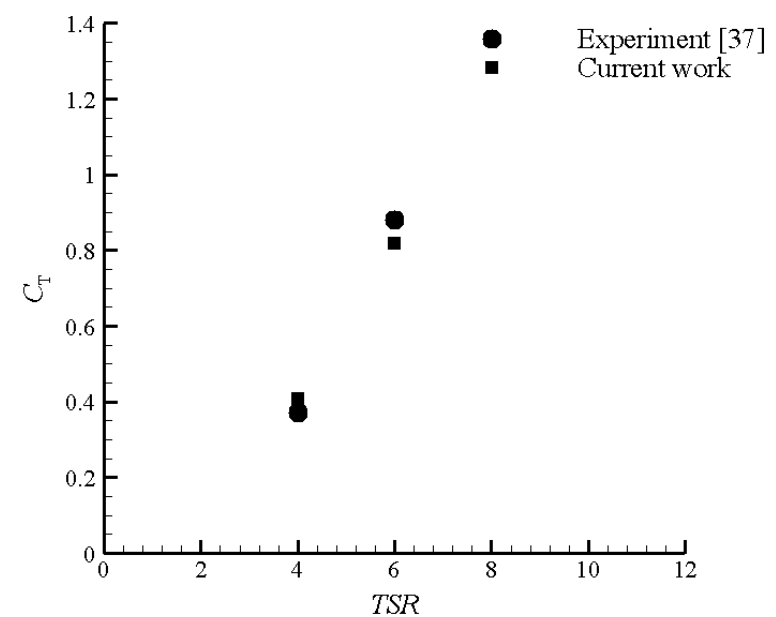

(b)

Fig. 6: $\mathrm{C}_{\mathrm{T}}$ comparison (a) single turbine, (b) two in-line turbines 


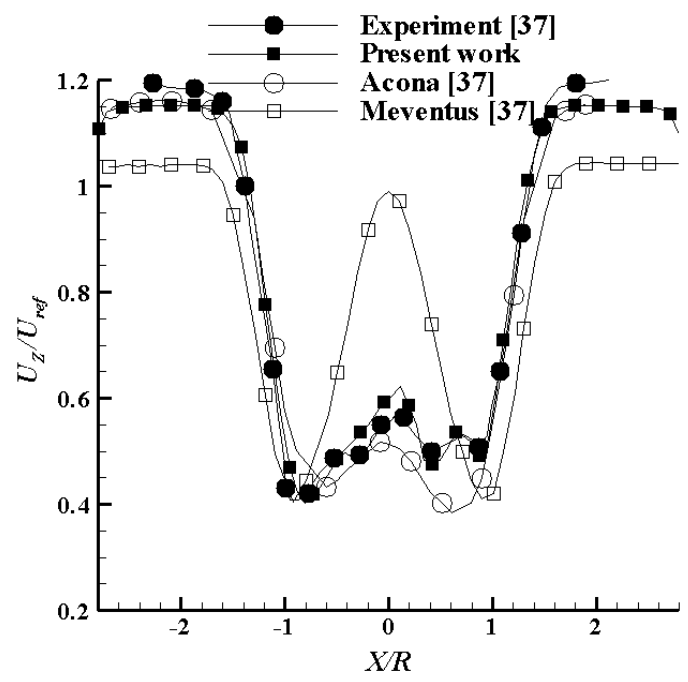

(a)

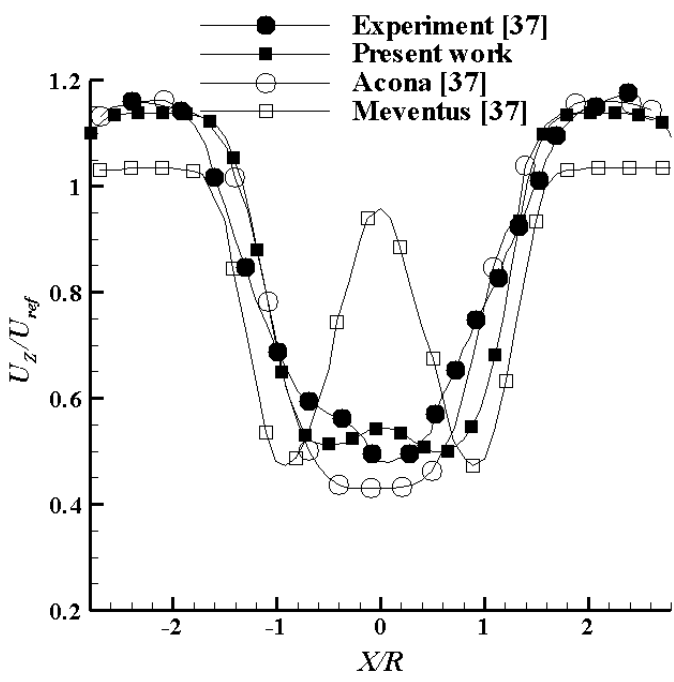

(b)

Fig. 7: Time averaged velocity profile along the horizontal lines centred at 1D (a) and 4D (b) in downstream of two in-line turbines

\subsection{The comparison of power coefficients}

As aforementioned, the clockwise blades are produced by mirroring the counter clockwise blades. Hence a single clockwise turbine is expected to have a same power coefficient as a single counter clockwise turbine under similar situations. Fig.8 shows the power coefficients at different rotational speeds for a single counter clockwise turbine and a single clockwise turbine. The acquired results revealed that power coefficients are almost identical at different rotational speeds in both setups. The performance of downstream wind turbine in a counter-rotating configuration is tested by decreasing the separation distances. The power coefficient of a downstream turbine has increased about $4 \%$ for counter-rotating configuration, when the separation distance is the same of a co-rotating configuration (3D). Power coefficient has increased about $7 \%$ by decreasing the separation distance to 1D in the counter-rotating configuration (Fig.9a). Fig.9.b shows the relative power coefficient of a counter-rotating configuration as a function of a co-rotating configuration at different separation distances. The results revealed operating the downstream turbine in the reverse direction of the upstream turbine will not only increase the power coefficient of the counter-rotating configuration, but will also significantly decrease the space required for this configuration. In other words, power efficiency is increased with no changes in the type of wind turbines and the layout of the two in-line setup. Hence, the overall performance of a wind farm could be improved considerably by the counter-rotating configuration. 


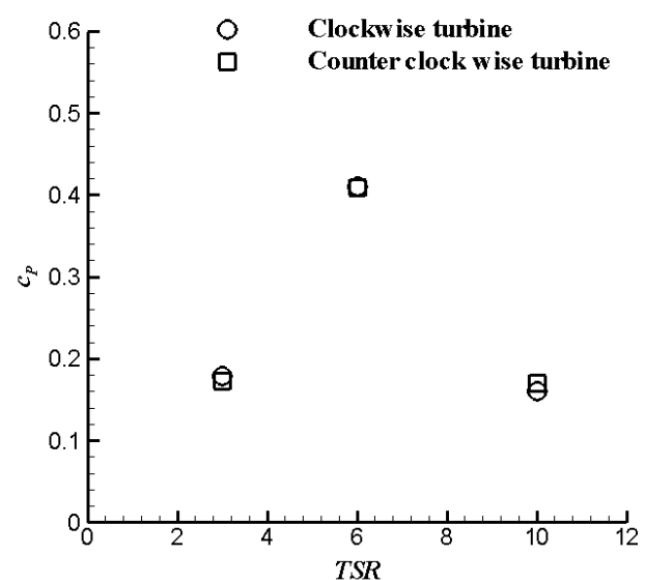

Fig. 8: Power coefficient comparison in a clockwise and counter clockwise turbine

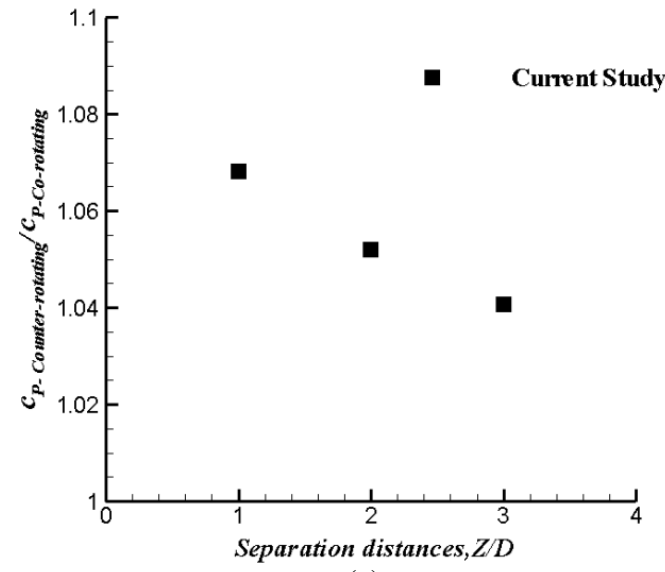

(a)

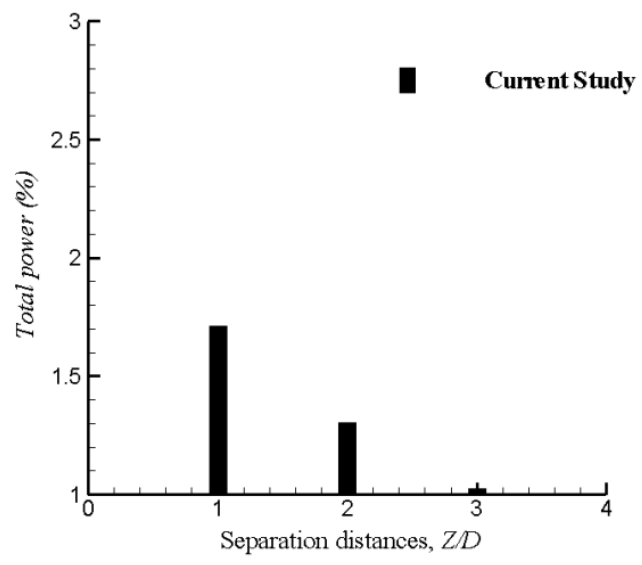

(b)

Fig. 9: (a) Power coefficient of downstream turbine in counter-rotating configuration. (b) Relative total power coefficient of counter-rotating configuration as a function of co-rotating configuration.

The oscillations of the power coefficient of a wind turbine under different situations are shown in Fig.10. The effect of rotation direction and separation distance on the power efficiency is the main objective of this part. Power coefficient shows a stable behaviour with time and slight fluctuation for a single wind turbine, Fig 8-a. The power curve of a downstream turbine has the same pattern for both co-rotating and counter-rotating configurations (Fig. 10.b, c). On the downstream turbine, a higher oscillation of power coefficient could be seen than a single turbine. Decreasing the separation distance has a significant effect on the power coefficient. The high oscillation of the power coefficient is observed in Fig. 10.d. More investigations are needed when this high level of fluctuations exists, in order to specify the exact location of encountering the vortices on downstream blades. So, the authors suggest a comprehensive study to extract and track the shed vortices of upstream turbine in the wake region. 


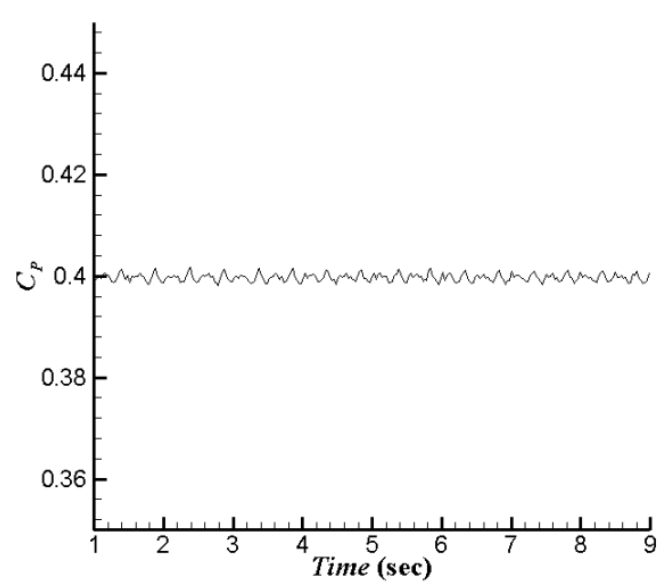

(a)

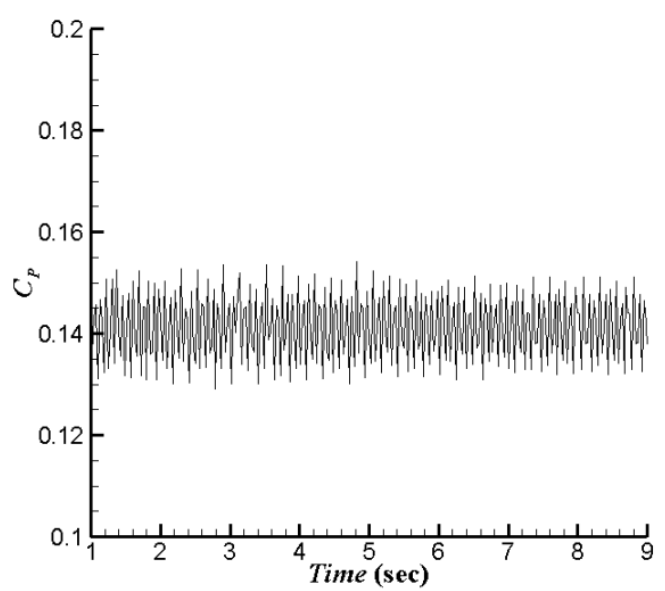

(c)

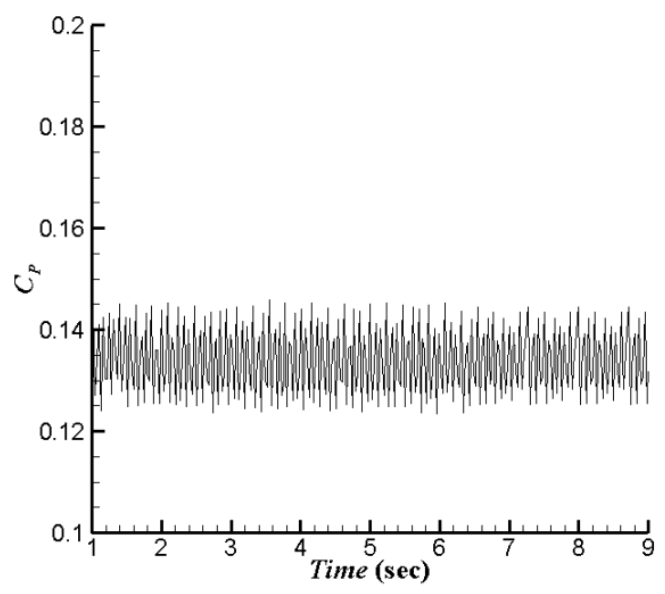

(b)

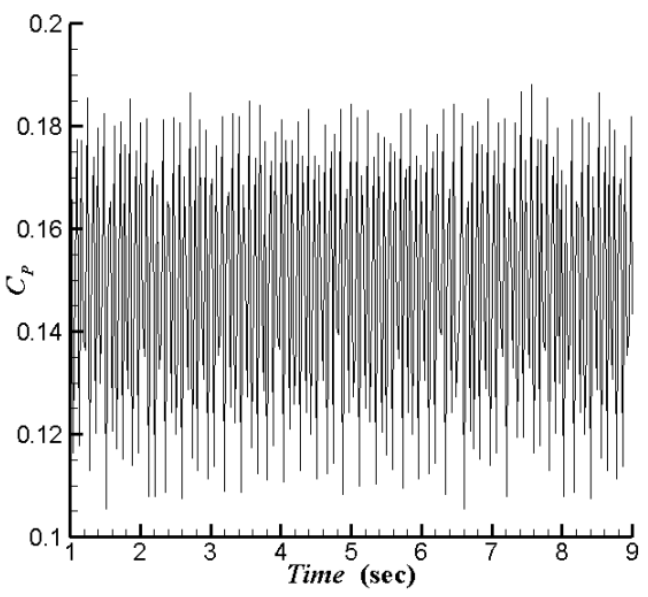

(d)

Fig. 10: oscillations of power coefficient $\left(C_{P}\right)(a)$ single wind turbine TSR=6. (b) Downstream turbine of co-rotating configuration at $\mathrm{TSR}=4$ (separation distance is $3 \mathrm{D}$ ). (c) Downstream turbine of counter-rotating configuration at $\mathrm{TSR}=4$ (separation distance is 3D). (c) Downstream turbine of counter-rotating configuration at TSR=4 (separation distance is 1D).

\subsection{Thrust coefficients}

Wind turbine blades are tolerated variable static and dynamic wind loads. Hence, the best mechanical design of wind turbines focuses on both types. The oscillations of thrust coefficients are shown in Fig.11 under different situations. Thrust coefficient is increased by increasing the rotational speeds for a single wind turbine. Thrust coefficient showed a higher level of oscillations for $\mathrm{TSR}=10$. The downstream wind turbine is exposed by lower flow velocity than the upstream wind turbine because of the velocity deficit in the wake region. Hence, the static wind load acting on the upstream turbine is higher than the downstream turbine, and the downstream turbine is experiencing higher levels of turbulence intensity than the upstream turbine. 


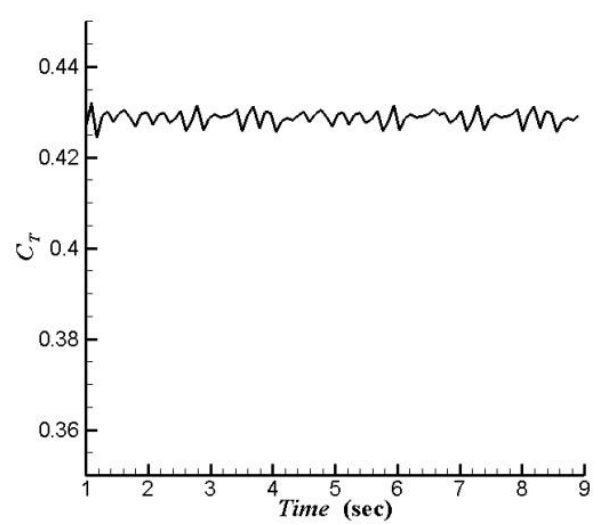

(a)

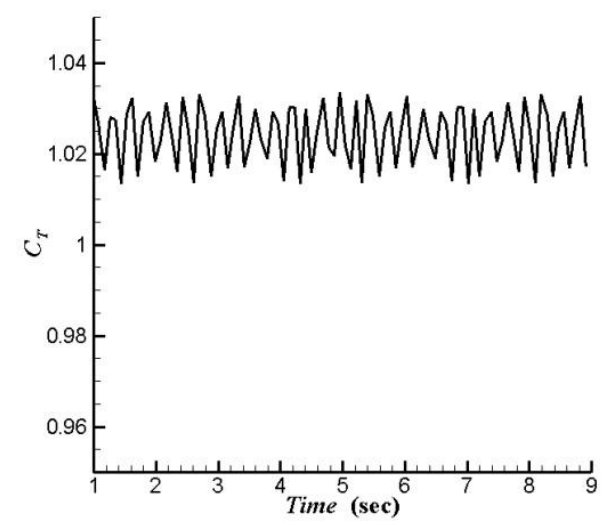

(c)

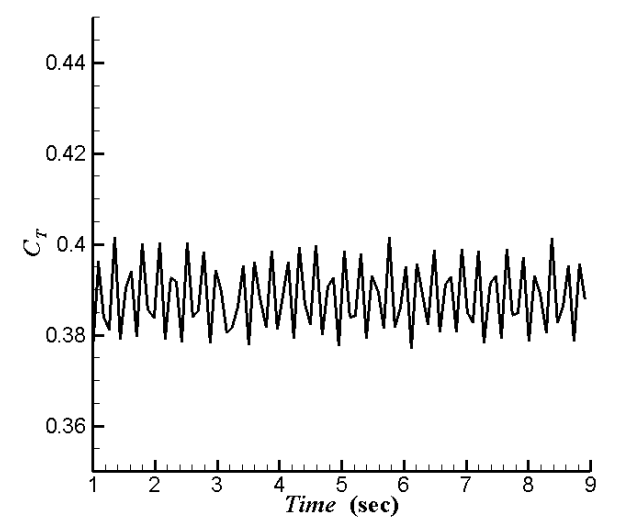

(e)

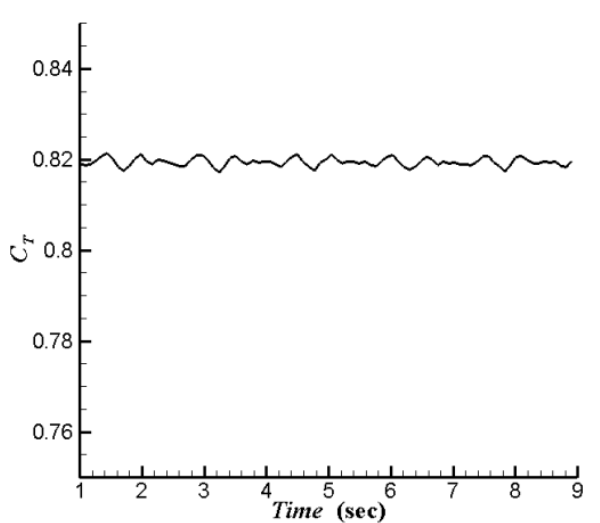

(b)

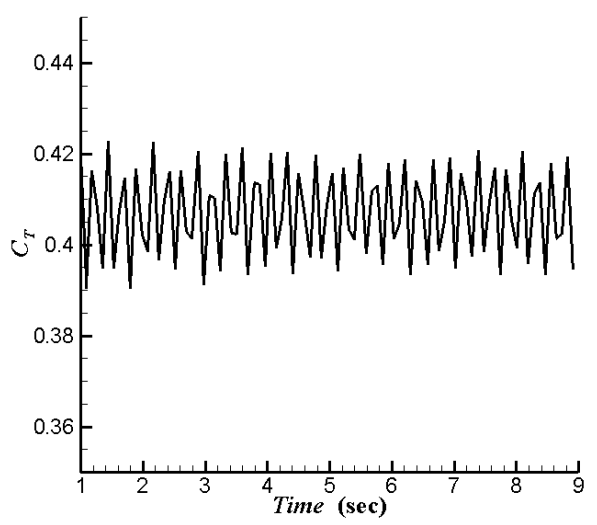

(d)

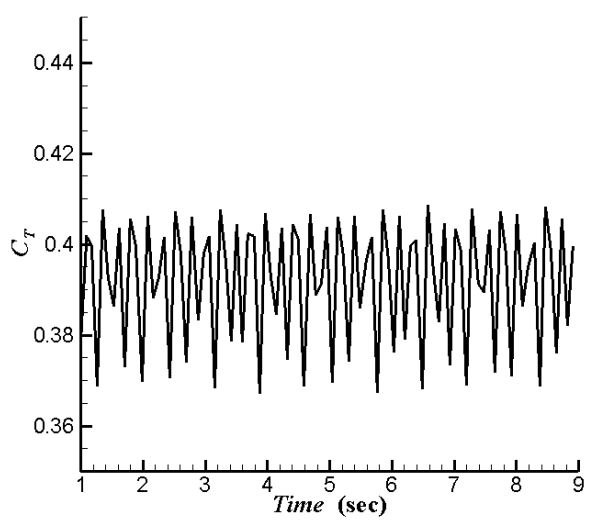

(f)

Fig. 11: oscillations of thrust coefficient $\left(C_{T}\right)$, (a) single wind turbine TSR=3, (b) single wind turbine TSR=6, (c) single wind turbine TSR=10, (d) downstream turbine of co-rotating configuration at TSR=4 (separation distance is 3D), (e) downstream turbine of counter-rotating configuration at TSR=4 (separation distance is $3 \mathrm{D}$ ), (f) downstream turbine of counter-rotating configuration at $\mathrm{TSR}=4$ (separation distance is $1 \mathrm{D})$.

For this reason, the oscillations of wind load on the downstream turbine blade (dynamic wind load) are much more than the upstream turbine. The results revealed the downstream turbine of counter-rotating configuration exposed lower static and dynamic wind loads (fig11.d, e) than the downstream turbine of co-rotating configuration. Therefore, the use of counter-rotating configuration without the change of separation distance has 
two major advantages: it improves the power coefficient of the two in-line turbines and lower wind loads act on the blades of downstream wind turbine. Fig.11.f showed that the dynamic wind loads are decreased by increasing the separation distance. However, the authors believed reducing the required space for the wind farm can be attractive and additional studies can be done on the material and the lifetime of blades.

\subsection{Turbulence structures}

A portion of wind energy is transferred to mechanical energy when the wind passes through the wind turbine, and the remaining part of energy becomes fluctuating energy in the wake region. The downstream wind turbine is affected by the wake of the upstream turbine. In fact, turbulence structures cause a significant dynamic wind load and vibrations in the downstream wind turbine. Hence a better understanding about the turbulence structures is inevitable if we are to optimise the efficiency of wind farms and decrease the dynamic wind loads. In a horizontal-axis wind turbine helical tip vortices are the main feature of this flow and play an important role in the wake region. The near wake region is affected by the number of blades, blade aerodynamics such as attached or stalled flows, 3-D effects, and tip vortices [26]. So, the study of turbulence structures could probably help to optimise the wind farm layout. In the present study, the tip local Reynolds number is $\operatorname{Re}_{c}^{t i p} \approx 10^{5}$ for the upstream turbine. This Reynolds number is higher than a full-scale wind turbine (at least one order of magnitude higher). It should be mentioned that the tip local Reynolds number has significant effects on the functional characteristics of wind turbines. For example, the maximum produced power from the wind turbine, which works at a low Reynolds number, is less than a similar wind turbine that works at a high Reynolds number [41]. Medici and Alfredsson [42] suggested that vortex shedding and turbulence structures in the near wake of wind turbines are independent of the tip local Reynolds number.

As mentioned above, turbulent structures should be revealed and investigated in order to study their effect on wind turbines. Vorticity cannot discriminate pure shearing motions from the actual swirling motions, so it is not an appropriate method to reveal turbulent structures. Hence, a precise vortex extraction method is required to reveal the vortical structures. Various tools and algorithms have been proposed and used by many investigators to identify vortices [43-51]. The $\lambda_{c i}$ method [51] is used to extract vortices in the present study. The mathematical description of $\lambda_{c i}$ described by Zhou et al [51]. By the position vector $\mathrm{r}$ in linear order the local velocity field around a point can be formulated as below:

$u(\mathrm{r}+\delta \mathrm{r})=\mathrm{u}(\mathrm{r})+\mathrm{D} \delta \mathrm{r}+\mathrm{o}\left(\|\delta r\|^{2}\right)$

where $\mathrm{D}$ is the velocity gradient tensor $(\nabla u)$. Its characteristic equation is expressed as:

$\lambda^{3}+P \lambda^{2}+Q \lambda+R=0$

Where $\quad Q=\frac{1}{2}\left[P^{2}-\operatorname{trace}(D D)\right], P=-\operatorname{trace}(D)=-\operatorname{div}(\boldsymbol{u})$ and $\quad R=\frac{1}{3}\left[P^{3}+3 P Q-\operatorname{trace}(D D D)\right]$

invariants of the velocity gradient tensor. The discriminant for this characteristic equation is

$\Delta=\left(\frac{1}{2} \tilde{\mathrm{R}}\right)^{2}+\left(\frac{1}{3} \tilde{\mathrm{Q}}\right)^{3}$ 
where $\tilde{R} \equiv R+\frac{2}{27} P^{3}-\frac{1}{3} P Q$ and $\tilde{\mathrm{Q}} \equiv Q-\frac{1}{3} P^{2}$. If $\Delta$ is positive, then the velocity gradient tensor has a real eigenvalue and a pair of conjugated complex eigenvalues. Moreover, the rotation rate is determined by $\lambda_{c i}$, and therefore, the imaginary part of the complex eigenvalue pair used as the local swirling strength of the vortex by Zhou et al. [51]. If $\lambda_{c i}$ is equal to zero, then there are no swirling motion or local circular streamline. The velocity gradient tensor in two-dimensional form can be expressed as:

$\nabla u=\left[\begin{array}{ll}\partial u / \partial x & \partial u / \partial y \\ \partial v / \partial x & \partial v / \partial y\end{array}\right]$

The discriminant for this characteristic equation is

$\Delta=\operatorname{trace}(\nabla \mathrm{u})^{2}-4 \operatorname{det}(\nabla \mathrm{u})$

$\lambda_{c i}$ is the imaginary part of conjugate pair of the complex eigenvalues of $\nabla \mathrm{u}$ as mentioned before and it is not zero when $\Delta>0$. Swirling strength has not designated the sense of the rotation since it is defined as the imaginary part of the complex eigenvalue of the local velocity-gradient tensor. The sign of swirling strength specified according to the sign of the spanwise vorticity as follows:

$\lambda_{c i}=\frac{\omega_{x}}{\left|\omega_{x}\right|} \lambda_{c i}$

where $\omega_{x}$ is the instantaneous fluctuating spanwise vorticity. Fig. 12 shows the swirling strength in the wake region of a single turbine for counter clockwise rotation and clockwise rotation setup. Zoom on top tip vortices are also shown. Top tip vortices are transported longer distances than hub/root vortices before they are dissipated. The effects of top tip vortices are not visible after 1.5D in the streamwise direction. Lu and PorteAgel [52] showed that top-tip vortices are not seen after 2D in the wake flow of a wind turbine. In both setups around the $Z=2.7$ to $Z=3.7$ six or seven vortices are seen. These features are not seen in the lower part of wind tunnels for tip vortices. Hence the frequency of top tip vortices has not changed under rotation direction. Hu et al. [26] found that the gap between two adjacent vortices be a function of the tip speed ratio. Tip vortices in the lower part of a wind tunnel are rapidly dissipated in the near wake regions for a single turbine. This could be due to the effect of tower and negligible velocity at the floor of the wind tunnel.

Fig. 13 shows the swirling strength in the wake region of co-rotating and counter-rotating configurations. Top tip vortices behind downstream turbine are extended more than a single turbine or upstream turbine. This issue could be due to superimposition of vortices that are shed from the upstream turbine. Tip vortices are extended in the $y$ direction behind the downstream turbine. The effects of upstream turbine are extracted by downstream turbine. Root vortices are only seen in the near wake region. These vortices lead to quick dissipation in all configurations $[53,54]$. In the far wake the effect of top tip vortices could not be clearly seen for a single turbine while for both two in-line configurations these effects are augmented. At the $Z=5$ to $Z=8$ these vortices are intensified. The tip vortices in the Counter-rotating configuration at the lower part of the wind tunnel are more extended than top tip vortices particularly in the y direction. 


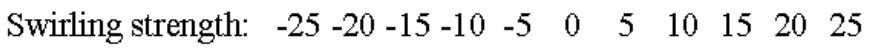
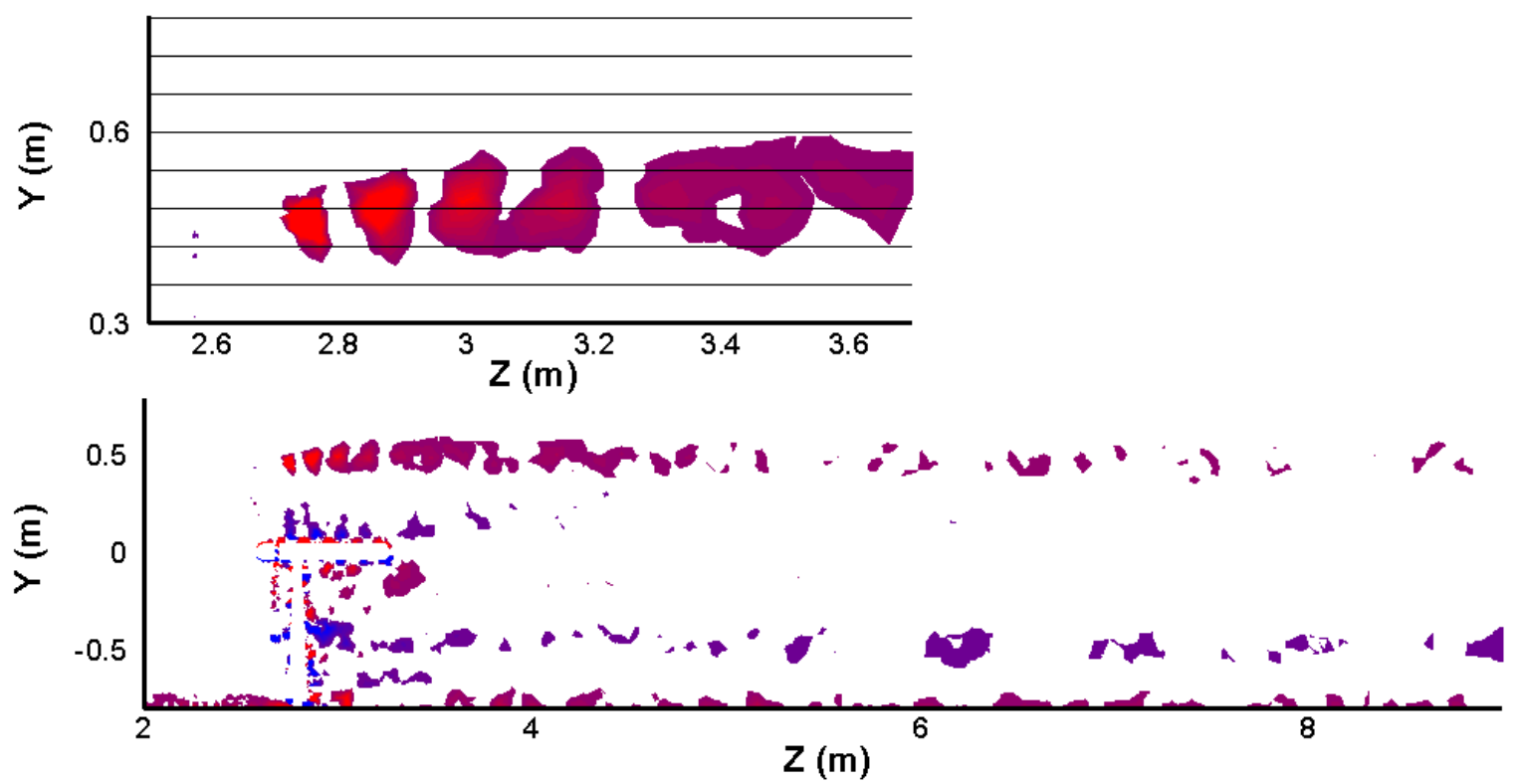

(a)

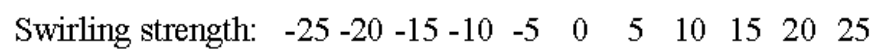
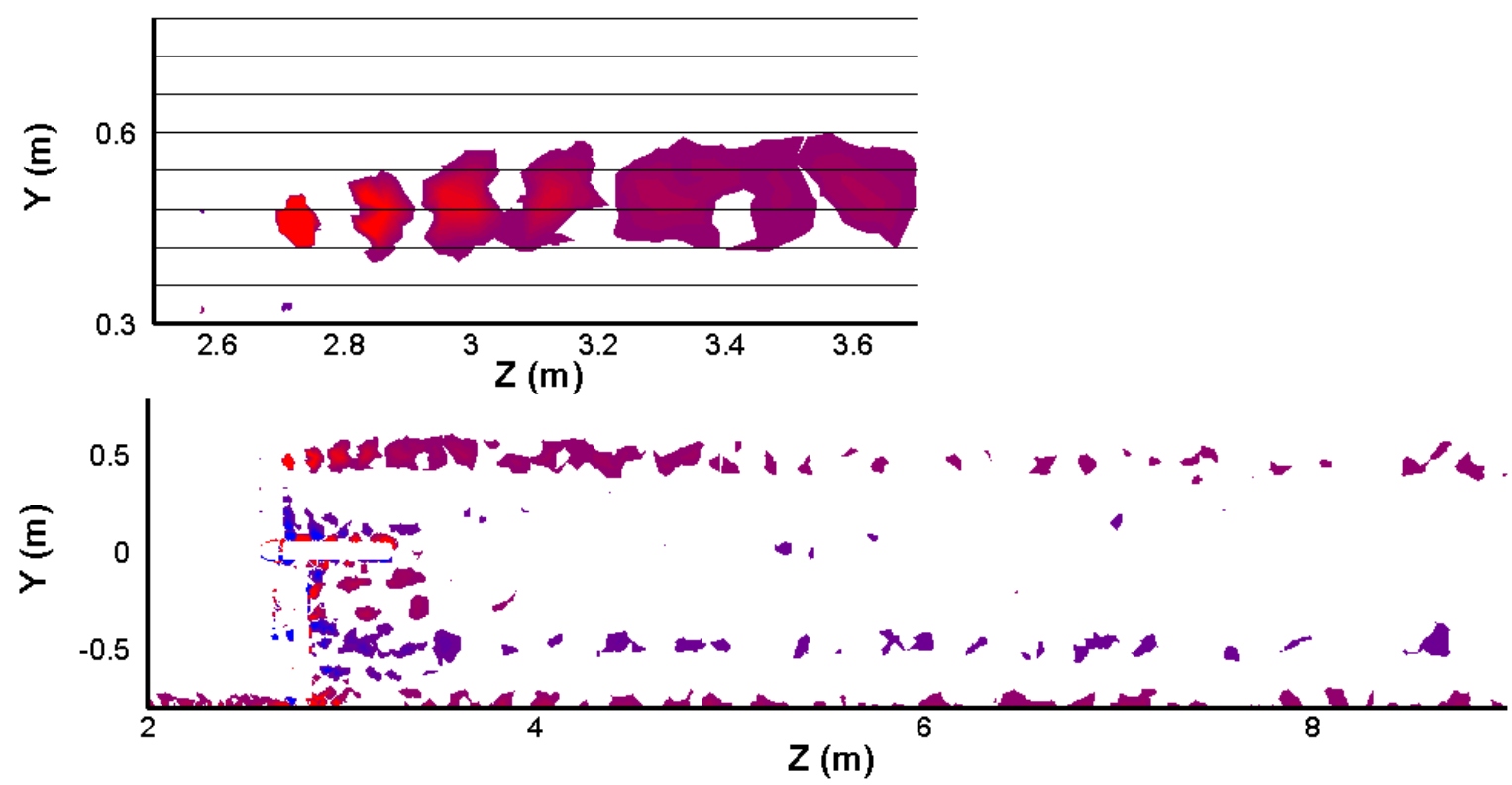

(b)

Fig. 12: swirling strength in the instantaneous velocity field (a) a counter clockwise turbine (b) a clockwise turbine

\subsection{Velocity profiles in the wake region}

The study of wake region is essential in order to estimate the velocity variations as fluid moves downstream of the wind turbine. For this purpose velocity contours are compared at different locations behind the wind turbine 
for co-rotating and counter-rotating configurations. The characteristics of the wake region reveal why a counterrotating configuration can harvest more wind energy than a co-rotating under similar conditions.

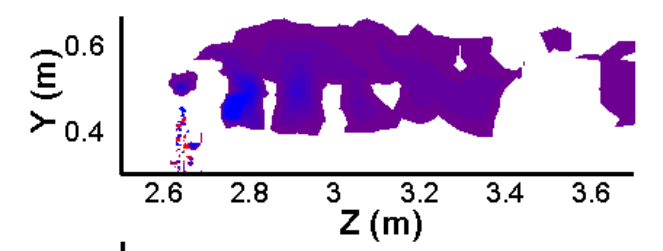

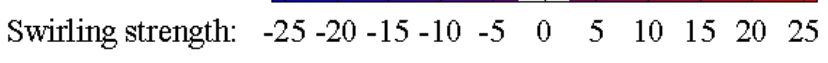
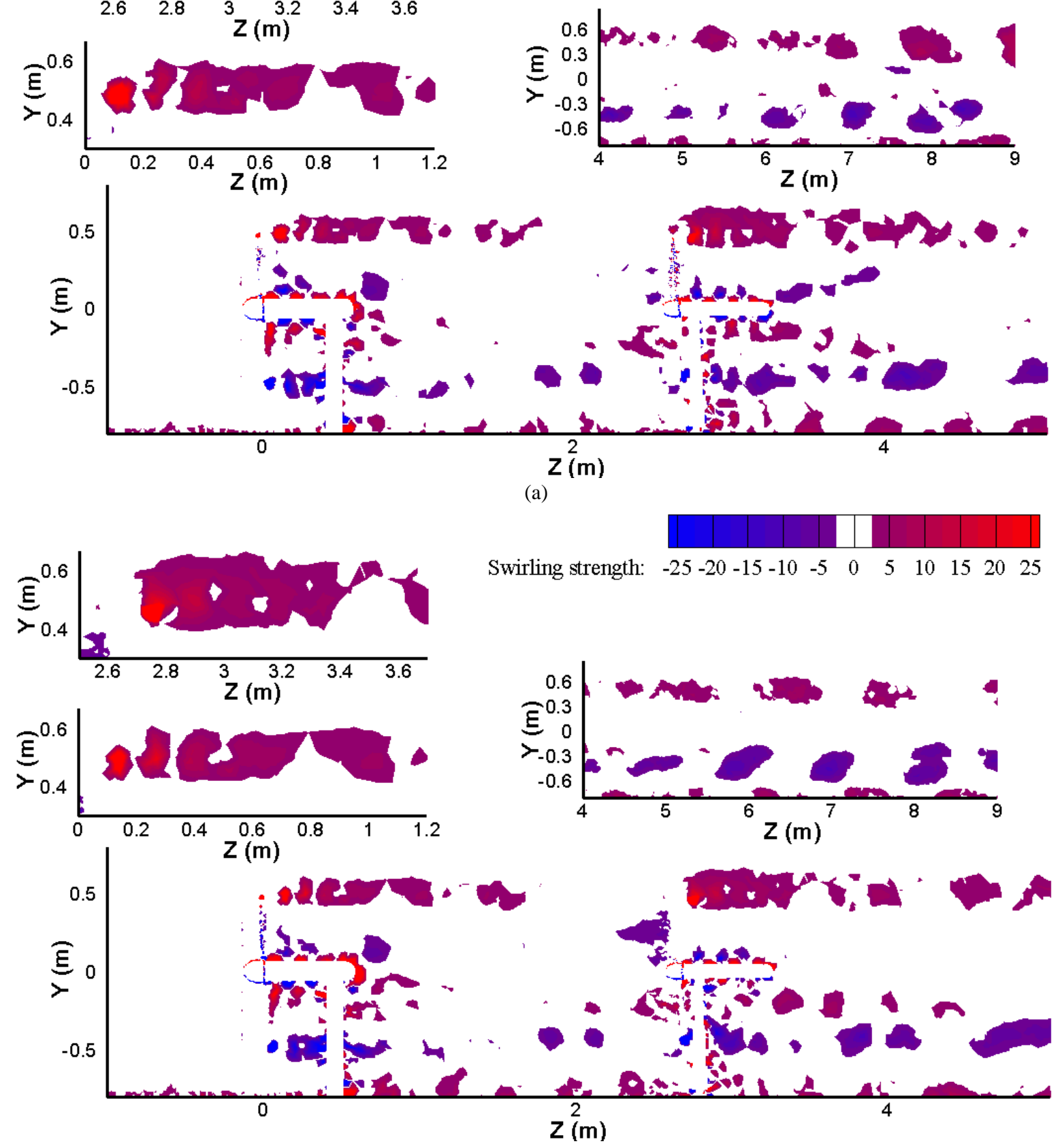

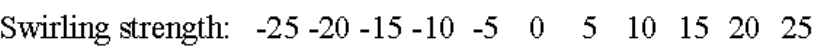

(a)

(b)

Fig. 13: swirling strength in the instantaneous velocity field (a) co-rotating configuration (b) counter-rotating configuration.

Fig 14 and 15 shows an overall view of time averaged velocity components in co-rotating and counter-rotating configurations in a central wind tunnel plane. A significant portion of wind energy carried by wind flow is harvested by the upstream wind turbines. So streamwise velocity is decreased and cross-stream and spanwise 
velocities are increased. The exerted torque by wind flow, which turns the blades, causes that downstream wake are rotating in the opposite direction of turbine blades $[12,26]$. In co-rotating configuration cross-stream velocity is reduced just behind the downstream turbine. Alternating positive and negative cross-stream velocity behind the downstream turbine of counter-rotating configuration shows an increase in energy production. The comparison of spanwise velocity in co-rotating and counter-rotating configurations only shows reduced effects of the root blade location in downstream direction. The streamwise velocity in both configurations showed an approximately similar pattern.
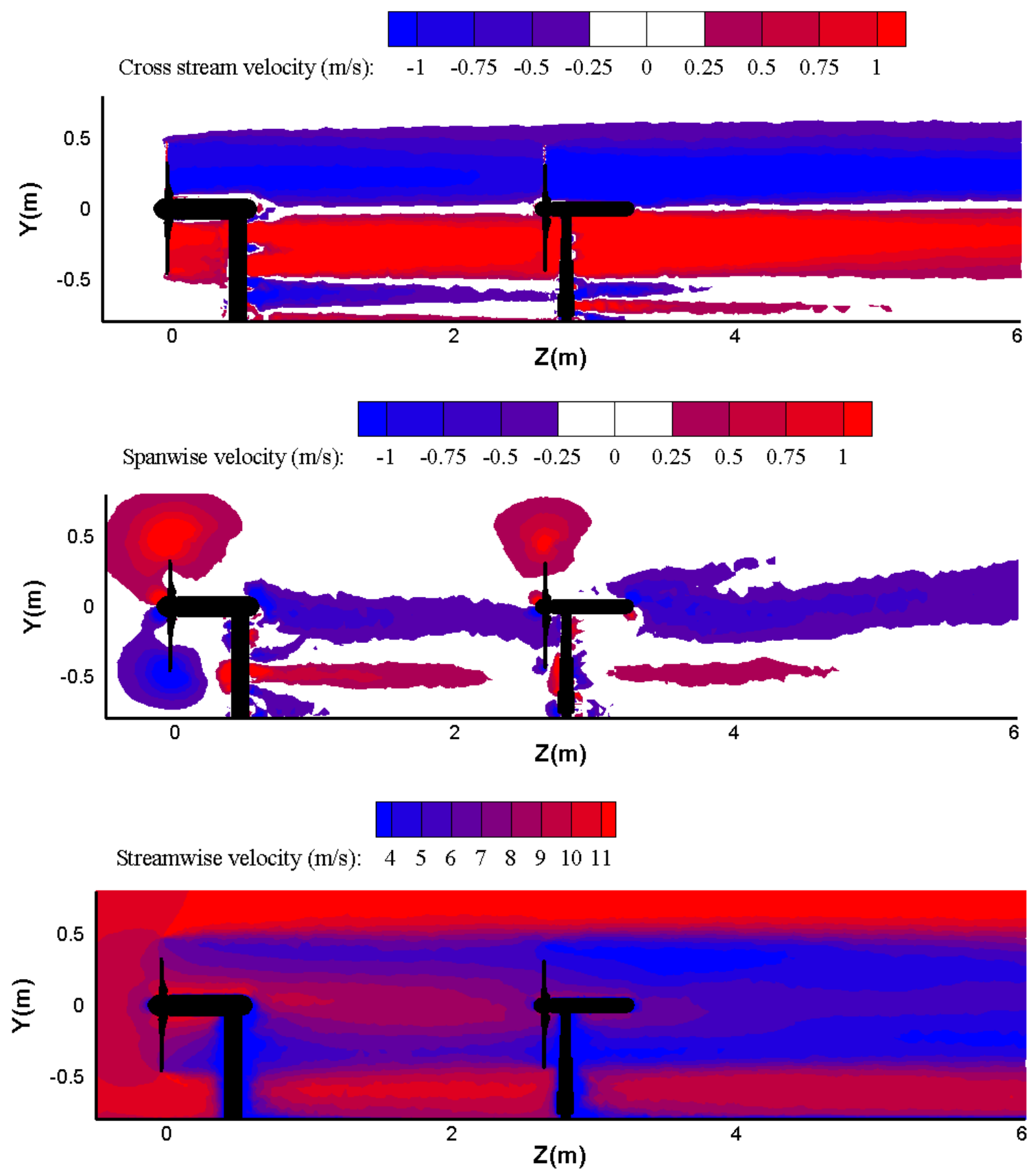

Fig.14: Time averaged velocity in the wake region of co-rotating configuration. (a) Cross stream velocity, (b) spanwise velocity, (c) streamwise velocity (third row) 

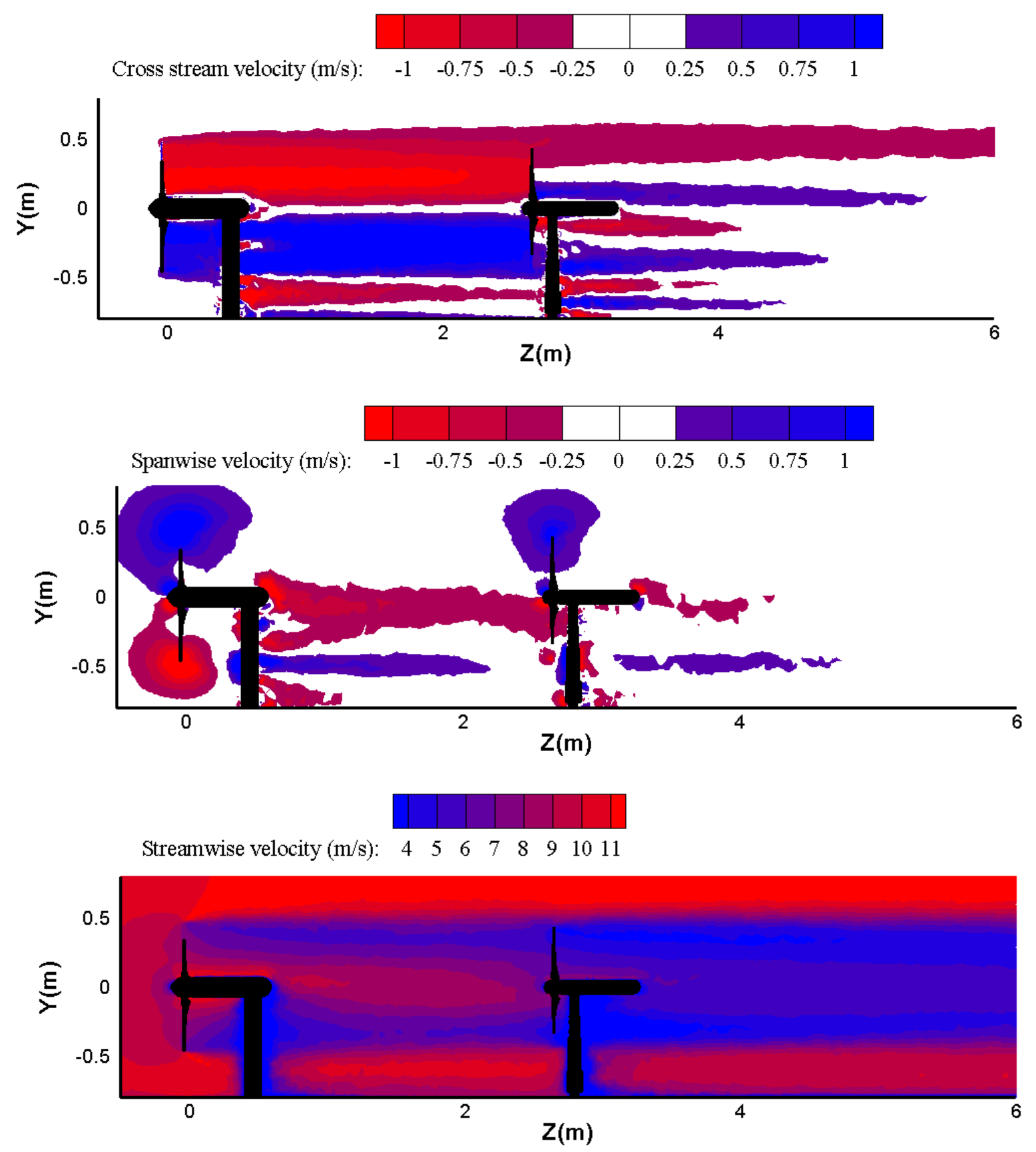

Fig.15: Time averaged velocity in the wake region of counter-rotating configuration. (a) Cross stream velocity, (b) spanwise velocity, (c) streamwise velocity (third row)

Fig.16 shows time-averaged cross-stream velocity contours in co-rotating and counter-rotating configurations at different locations behind wind turbines (velocity vectors are super-imposed). The rotation of the wake is seen in cross-stream velocity contours and velocity vectors. The positive value of the cross-stream velocity contours shows the rotation is toward the inside while negative value is outward in an $x-y$ plane. At $Z / D=4$ in the downstream direction of the wind turbine the cores stream velocity is contracted and take over a smaller area. By moving down stream at $\mathrm{Z} / \mathrm{D}=7$ the cores stream velocity with this level of intension are vanishing, but two cores for each area with lower intensity are produced (Fig.16a). The velocity distribution become smoother as wake flow moved in the downstream direction in the swept area. The cross stream velocity is decreased due to the turbulence mixing and the wake is recovered by its moving downstream for both configurations. A regular 
pattern is not seen in the wake region of counter-rotating configuration. This could be due to the wake interaction between the upstream and downstream turbines. The rotation direction of flow is a function of blade rotation direction. So in a clockwise turbine the positive region is changed by the negative region of cross-stream velocity. In counter-rotating configuration the flow field behind the upstream turbine has the same direction of rotation direction of the downstream turbine (Fig.16b). For this reason, in a counter-rotating configuration the downstream turbine harvests more wind energy than a co-rotating configuration. Moreover, in counter-rotating configuration smaller amount of cross-stream velocity than co-rotating configuration can attest to this claim. The distribution of cross stream velocity in counter-rotating configuration shows a different pattern. In $\mathrm{Z} / \mathrm{D}=1$ two positive and two negative regions are seen (Fig.16b). In other words, the wake of the downstream turbine is penetrated into the wake of upstream turbine and produced this flow distribution. A portion of cross stream velocity that is produced by the upstream turbine is absorbed via a downstream turbine. The effect of the tower has the same pattern in both setups in downstream direction. This effect disappeared after $\mathrm{Z} / \mathrm{D}=1$.

Fig. 17 shows time averaged spanwise velocity contours in co-rotating and counter-rotating configurations at different locations behind the wind turbines (velocity vectors are super-imposed). The spanwise velocity of both configurations has a similar pattern of the cross-stream velocity. In both configurations the spanwise velocity is decreased by increasing downstream distance. Spanwise velocity of the downstream turbine is penetrated to the spanwise velocity of the upstream turbine that (Fig.17b). More white zones in the swept area of a counterrotating configuration is the reason for harvesting more energy from the wind flow compared with co-rotating configuration under similar conditions (Fig. 17b).

Fig.18 shows ensemble-averaged streamwise velocity contours (velocity vectors are super-imposed) in corotating and counter-rotating configurations at different locations behind the wind turbine. Wake effects are decreased as moving in the downstream direction of the wind turbine. A relatively symmetrical wake distribution can be seen behind the wind turbine at $\mathrm{Z} / \mathrm{D}=1$ of both configurations. A nearly symmetric region has also been observed by Chamorro and Porte-Agel [55]. As wake moves in the downstream direction, wake region is compressed in the y direction for co-rotating configuration while in counter-rotating configurations wake region is compressed in the $\mathrm{x}$ direction. Streamwise velocity decreases from the centre of the wake (hub location) to the edge of swept area (tip of the blade). This issue could be due to the tip of the blades that extract more energy from the wind than the root of the blades. Xie et al. [56] also divided the swept area into low and high efficiency areas like the present work. In other words, the blade speed at the tip is much higher than the root and the energy extraction is affected by the blade speed. The development of streamwise velocity contours in the wake region of both configurations is almost identical. In other words, higher efficiency in the counter-rotating configuration has not been under the influence of streamwise velocity, while cross stream and spanwise velocity are changed greatly. As a result, the best power coefficient is due to the lateral velocities. Therefore, the power coefficient is increased by decreasing the separation distance in a counter-rotating configuration. This assumption is also confirmed by the study of tip vortices.

Fig. 19 shows time averaged cross-stream velocity profile along a horizontal line, centred at two separation distances in counter-rotating configuration. By decreasing the separation distances cross-stream velocity is decreased. 

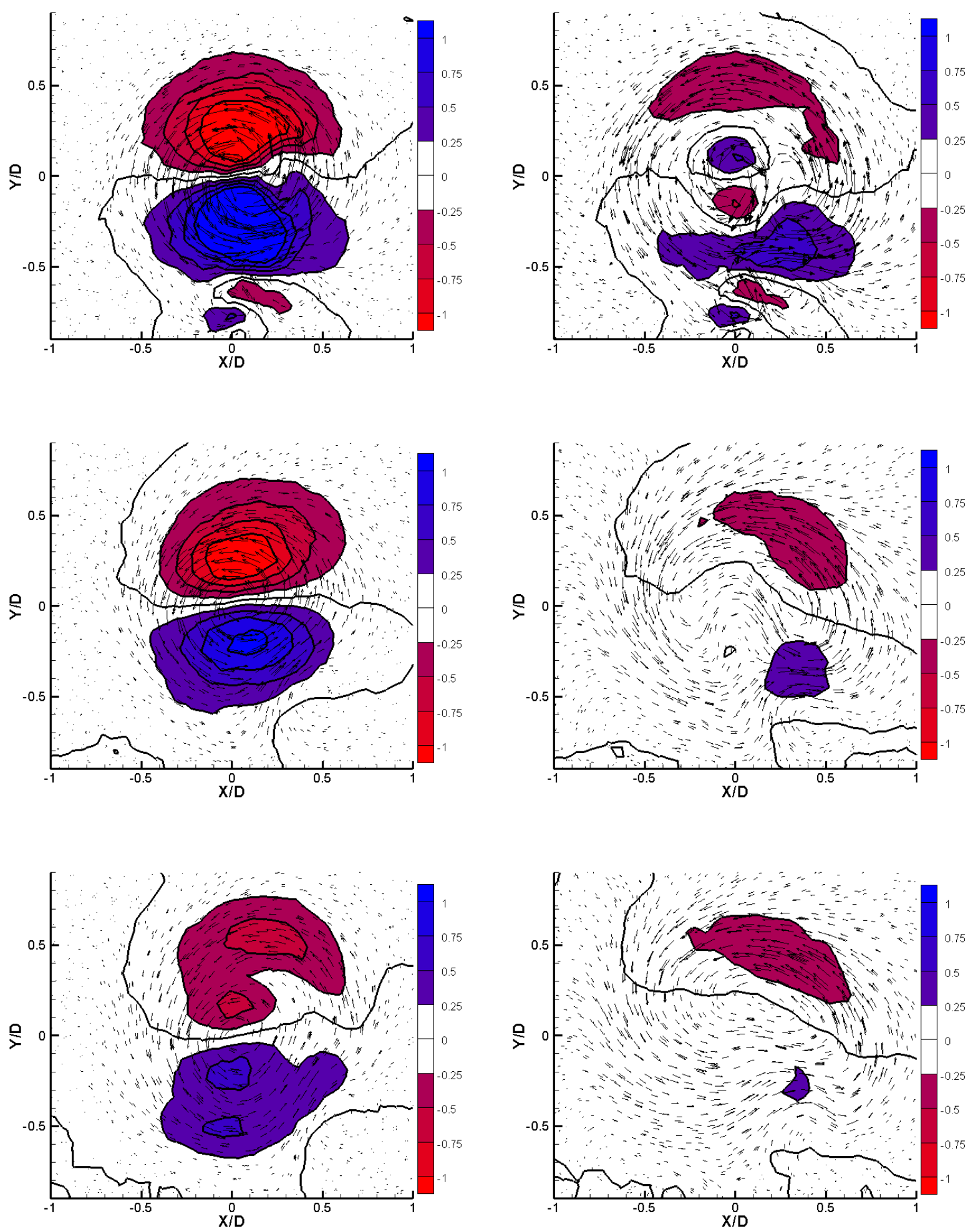

(a)

(b)

Fig. 16: Contours of the ensemble-averaged cross-stream velocity (velocity vectors are super-imposed) in spanwise planes at $\mathrm{z} / \mathrm{D}=1$ (first row); $\mathrm{z} / \mathrm{D}=4$ (second row) and $\mathrm{z} / \mathrm{D}=7$ (third row). (a) Co-rotating configuration (left column); (b) counterrotating configuration (right column). 

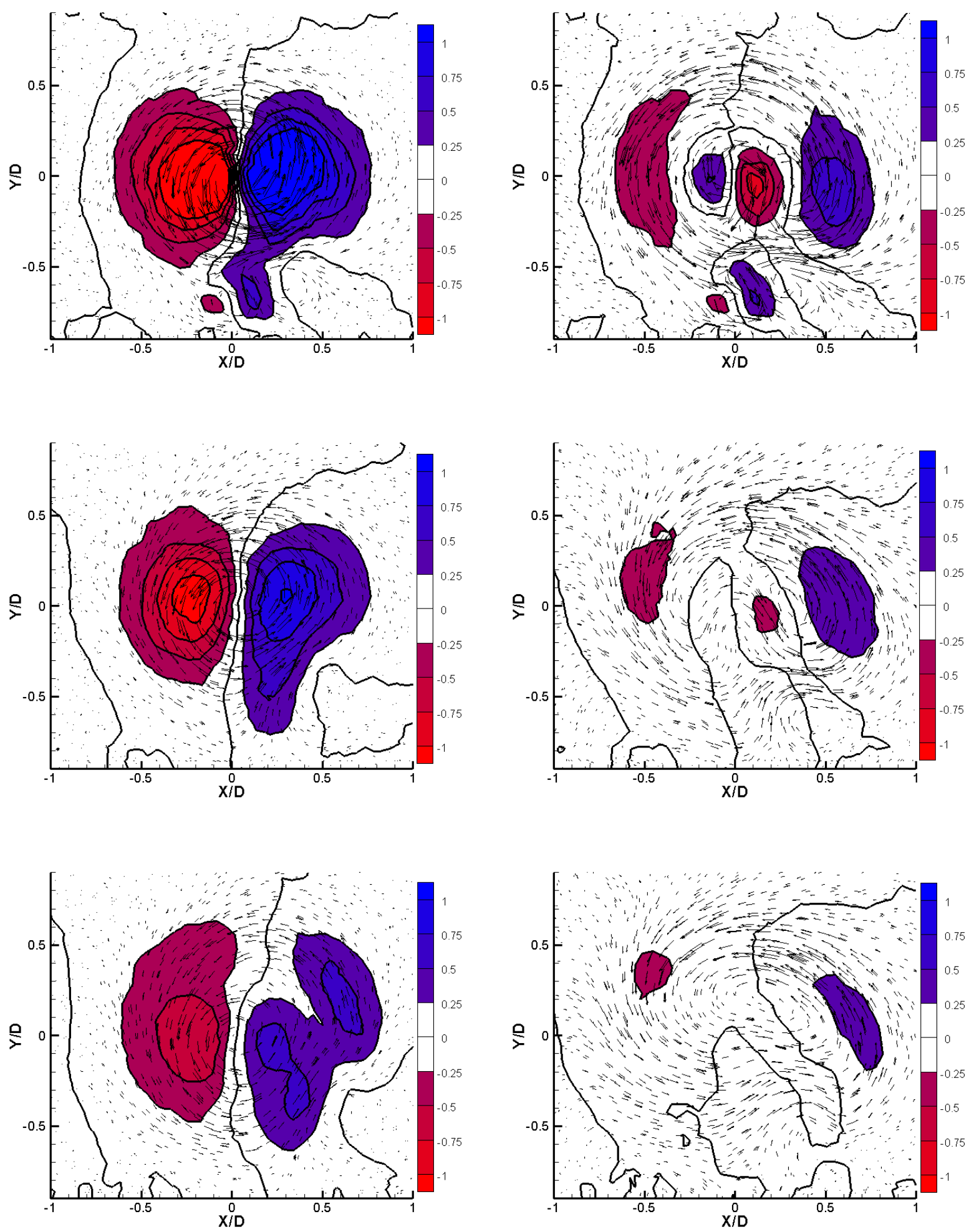

(a)

(b)

Fig. 17 Contours of the ensemble-averaged spanwise velocity (velocity vectors are super-imposed) in spanwise planes at $\mathrm{z} / \mathrm{D}=1$ (first row); $\mathrm{z} / \mathrm{D}=4$ (second row) and $\mathrm{z} / \mathrm{D}=7$ (third row). (a) Co-rotating configuration (left column); (b) counterrotating configuration (right column). 

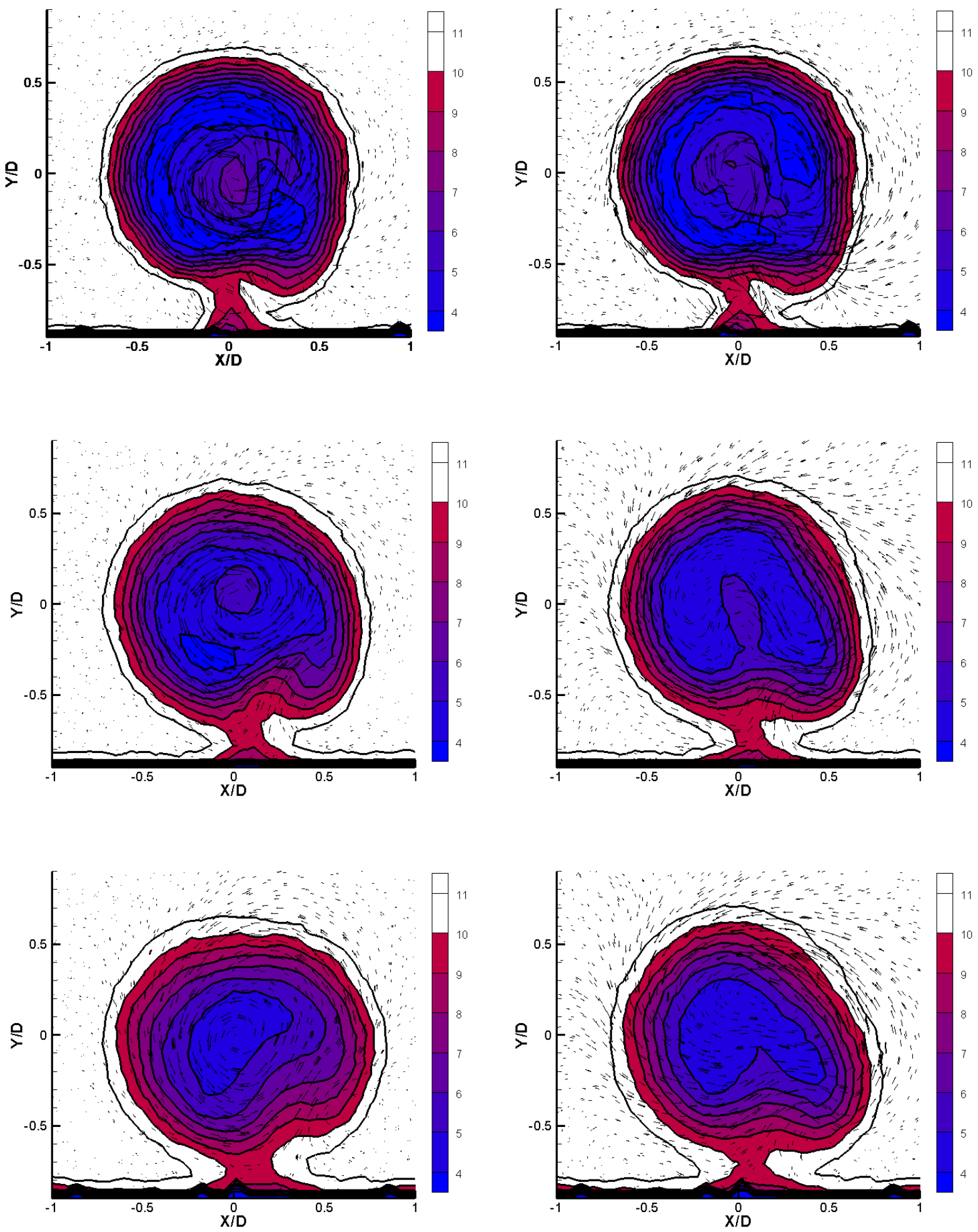

(a)

(b)

Fig. 18 Contours of the ensemble-averaged streamwise velocity (velocity vectors are super-imposed) in spanwise planes at $\mathrm{z} / \mathrm{D}=1$ (first row); $\mathrm{z} / \mathrm{D}=4$ (second row) and $\mathrm{z} / \mathrm{D}=7$ (third row). (a) Co-rotating configuration (left column); (b) counterrotating configuration (right column). 


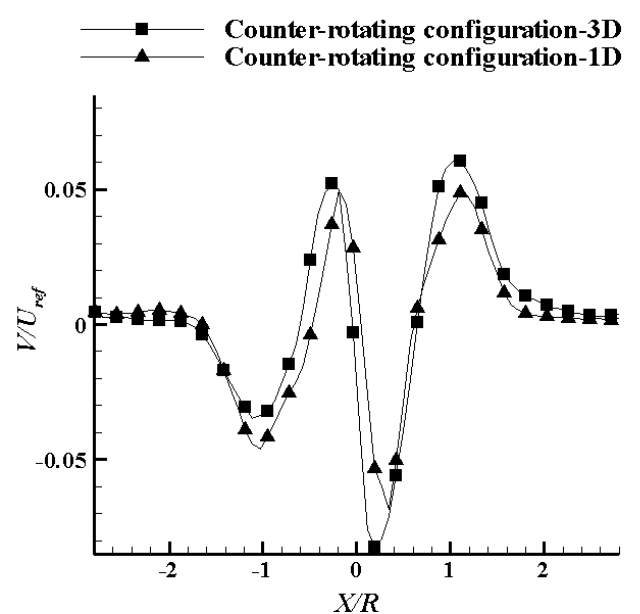

(a)

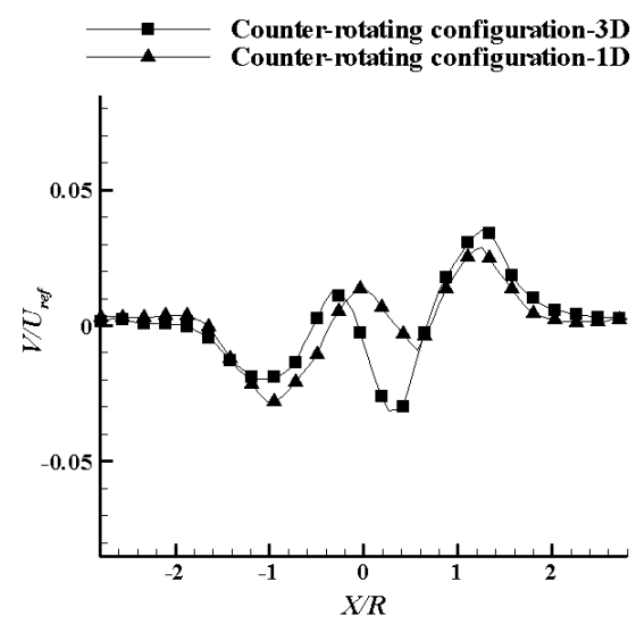

(b)

Fig. 19: Time averaged cross stream velocity profile along the horizontal lines centered at two separation location of a counter-rotating configuration (a) 1D (b) 4D in downstream of turbines

\subsection{Turbulence Intensity}

Turbulence structure, and predominantly turbulence intensity, is defined as a criterion of fatigue loads on a downstream wind turbine. So the study of spatial distribution of turbulence intensity could be valuable to minimise the effect of fatigue loads on downstream wind turbines and optimise the layout of the wind farm. Fig.20 shows turbulence intensity along a horizontal line, centred in one location after the inlet of the wind tunnel and four locations behind wind turbines of co-rotating and counter-rotating configurations.

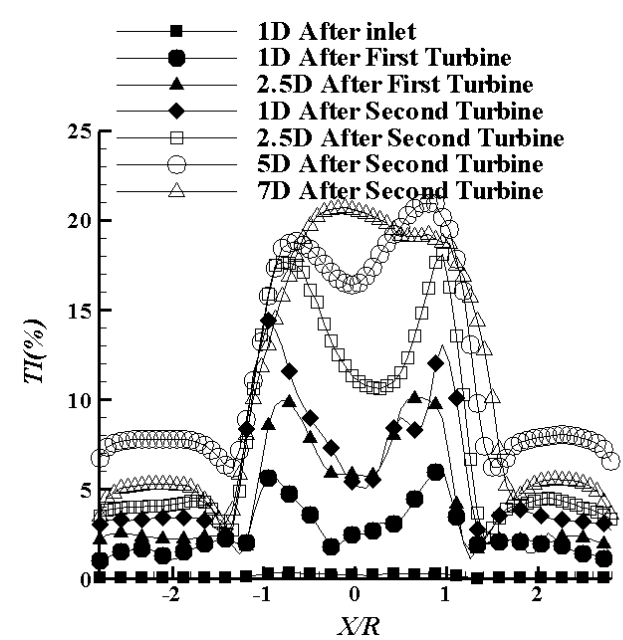

(a)

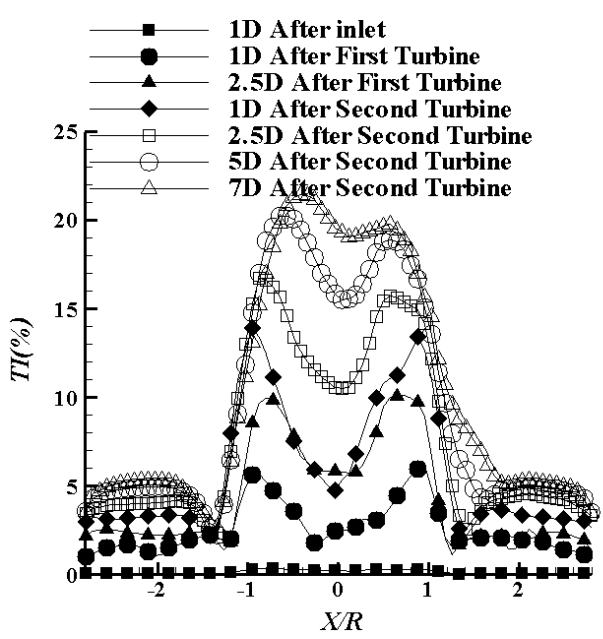

(b)

Fig.20 Ensemble averaged streamwise turbulence intensity along the horizontal lines centred at different locations (a) corotating configuration, (b) counter-rotating configuration.

The comparison of turbulence intensity before and after the wind turbines confirmed that the downstream turbine experienced more turbulence intensity than the upstream turbine. Hence the fatigue lifetime of downstream turbine is decreased. Turbulence intensity is increased by increasing downstream distance in both configurations. 
Tip vortices are important characteristic in the near wake, as they are shed from the blade tip and moved in the downstream direction and finally dissipated. The recovery of turbulence intensity is happening slower than the streamwise velocity in the wake region [11]. These vortices are the main source of noise and vibration of the blades. Two local maximum of turbulence intensity is observable nearby the $X / R= \pm 0.9$ in both configurations. This issue could be due to the tip vortices. The effects of these points are reduced by moving in a downstream direction. These local maximums are dissipated at 7D in co-rotating configuration while in counter-rotating configuration these points can still be seen. A local minimum is visible at the centre of the rotor in turbulence intensity profile for both configurations. This event could be due to vortices that shed from the hub and root and slower speed (near to zero) at the root. The results revealed that the turbulence intensity has a similar pattern in both configurations.

\section{Conclusion}

The present study focuses on the effects of the rotation direction of the downstream turbine on the power coefficient of two in-line turbines. LES has been employed to have the velocity domain and investigate the wake region in co-rotating and counter-rotating configurations. The acquired results are in good agreement with the experimental data reported in literatures. For a single turbine the power coefficient is almost the same in a clockwise turbine and a counter-clockwise turbine at different rotational speeds. Power coefficient is increased by about $4 \%$ for the downstream turbine in counter-rotating configuration than co-rotating configuration with the same separation distance. The study of the thrust coefficient showed both static and dynamic wind loads decrease in a downstream turbine of counter-rotating configuration. The use of counter-rotating configuration without the change of separation distance (3D) has two major advantages: it improves the power coefficient of the two in-line wind turbines and lower wind loads act on the blade of the downstream wind turbine. The power efficiency of downstream turbine is increased by about $7 \%$ by decreasing the separation distance to 1D. As the fluctuation of the power curve is so high, the exact location of encountering the shed vortices on the downstream turbine should be determined to improve the power efficiency. In fact, operating the wind turbines in counterrotating configuration has two important effects: first, it improves the power coefficient of two in-line wind turbines. Second, the space required is also decreased for the given two in-line configuration. Dynamic wind loads on the downstream turbine are increased by decreasing the separation distance. However, the authors believe that space reduction and power efficiency of the wind farms are interesting subjects. So, more studies are reasonable, as they will illuminate how to increase the strength and lifetime of the blade.

Tip vortices are the main feature of a horizontal-axis wind turbine. In the present work the $\lambda_{c i}$ method is employed to visualise turbulence structures in the wake region. The effects of top tip vortices are not visible after 1.5 D in a single wind turbine in both counter-clockwise and clockwise turbines. Tip vortices in the lower part of the wind tunnel are sooner dissipated than top tip vortices. This could be due to the tower effect and also negligible velocity at the floor of the wind tunnel. Top tip vortices are more extended in the y direction in both two in-line configurations than a single turbine. This could be due to the effect of upstream turbines superimposed on the vortices of a downstream turbine. In the far wake region, the effect of top tip and bottom vortices are visible in both two in-line configurations, while these vortices are not seen in a single turbine. 
Wake region is influenced by the turbines that are installed at an upstream direction. By moving in the downstream direction, the velocity field is changed and the effect of wake is decreased. The flow is rotated faster, closer to the centre than the corners in the wake region for co-rotating configuration. The velocity filed became smoother in the swept area by moving in a downstream direction. An irregular pattern is created by the interaction between the wake of upstream and downstream turbine wake in a counter-rotating configuration. In this configuration the rotation direction of a downstream turbine is the same rotation direction of upstream wake flow. This is why the downstream turbine in counter-rotating configuration can harvest more energy than a corotating configuration. In a counter-rotating configuration the wake of the downstream turbine is entered in the wake of the upstream turbine and created two positive and negative areas. The distribution of streamwise velocity is relatively symmetrical at $\mathrm{Z} / \mathrm{D}=1$ in the downstream direction of a wind turbine in both configurations. By moving in the downstream direction, this symmetrical streamwise velocity is compressed in y direction for co-rotating configuration, while it is compressed in the $\mathrm{x}$ direction for counter-rotating configuration. Higher velocity deficits in tip blade related to the hub/root blade locations could be due to the tip vortices. Moreover, this velocity deficit could be also the effect of blade speed in that it is much higher than the root. As a result, energy extraction is affected by the blade speed. Streamwise velocity is almost identical at different locations in both configurations. In other words, better performance of the downstream turbine in counter-rotating configuration is due to the lateral velocities. The effect of flow rotation and tip vortices is much higher in the near wake. The efficiency of a two in-line turbines is increased by decreasing the distance between two consecutive turbines in a counter-rotating configuration.

The comparison of turbulence intensity showed that it is increased by moving in the downstream direction in both co-rotating and counter-rotating configurations. The local maximum value of turbulence intensity at the blade tip locations could be due to the effect of tip vortices. A local minimum of turbulence intensity at the hub location could be due to weak vortices that shed from hub/root and blade speed that are near to zero at this point. The mentioned effects are decreased by travelling in a downstream direction. The acquired results show that turbulence intensity is slightly different in both configurations.

\section{Acknowledgements}

The authors would like to acknowledge the Department of Energy and Process Engineering of the Norwegian University of Science and Technology for providing all the geometry data.

\section{References}

[1] Chen WH, Chen CY, Huang CY, Hwang CJ. Power output analysis and optimization of two straight-bladed vertical-axis wind turbines. Appl Energy 2017;185:223-32. doi:10.1016/j.apenergy.2016.10.076.

[2] Chehouri A, Younes R, Ilinca A, Perron J. Review of performance optimization techniques applied to wind turbines. Appl Energy 2015;142:361-88. doi:10.1016/j.apenergy.2014.12.043.

[3] Danao LA, Edwards J, Eboibi O, Howell R. A numerical investigation into the influence of unsteady wind on the performance and aerodynamics of a vertical axis wind turbine. Appl Energy 2014;116:111-24. doi:10.1016/j.apenergy.2013.11.045.

[4] Pagnini LC, Burlando M, Repetto MP. Experimental power curve of small-size wind turbines in turbulent urban environment. Appl Energy 2015;154:112-21. doi: 10.1016/j.apenergy.2015.04.117.

[5] Roy S, Saha UK. Wind tunnel experiments of a newly developed two-bladed Savonius-style wind turbine. Appl Energy 2015;137:117-25. doi: 10.1016/j.apenergy.2014.10.022. 
[6] Saeidi D, Sedaghat A, Alamdari P, Alemrajabi AA. Aerodynamic design and economical evaluation of site specific small vertical axis wind turbines. Appl Energy 2013;101:765-75. doi: 10.1016/j.apenergy.2012.07.047.

[7] Al-Hadhrami LM. Performance evaluation of small wind turbines for off grid applications in Saudi Arabia. Energy Convers Manage 2014;81:19-29. doi: 10.1016/j.enconman.2014.01.064.

[8] Delarue ED, Luickx PJ, D'haeseleer WD. The actual effect of wind power on overall electricity generation costs and CO2 emissions. Energy Convers Manage 2009;50(6):1450-6. doi: 10.1016/j.enconman.2009.03.010.

[9] Li Y, Paik KJ, Xing T, Carrica PM. Dynamic overset CFD simulations of wind turbine aerodynamics. Renew Energy 2012;37:285-98. doi:10.1016/j.renene.2011.06.029.

[10] Grant I, Parkin P, Wang X. Optical vortex tracking studies of a horizontal axis wind turbine in yaw using laser-sheet, flow visualisation. Exp Fluids 1997;23:513-9. doi:10.1007/s003480050142.

[11] Vermeer LJ, Sørensen JN, Crespo A. Wind turbine wake aerodynamics. Prog Aerosp Sci 2003;39:467-510. doi:10.1016/S0376-0421(03)00078-2.

[12] Zhang W, Markfort CD, Porté-Agel F. Near-wake flow structure downwind of a wind turbine in a turbulent boundary layer. Exp Fluids 2012;52:1219-35. doi:10.1007/s00348-011-1250-8.

[13] Park J, Law KH. Layout optimization for maximizing wind farm power production using sequential convex programming. Appl Energy 2015;151:320-34. doi:10.1016/j.apenergy.2015.03.139.

[14] Wu Y T, Porté-Agel F. Simulation of turbulent flow inside and above wind farms: Model validation and layout effects. Boundary-Layer Meteorology, 2013;146:181-205.doi: 10.1007/s10546-012-9757-y.

[15] Gao X, Yang H, Lu L. Optimization of wind turbine layout position in a wind farm using a newly-developed twodimensional wake model. Appl Energy 2016;174:192-200. doi: 10.1016/j.apenergy.2016.04.098.

[16] Stevens RJAM, Gayme DF, Meneveau C. Large eddy simulation studies of the effects of alignment and wind farm length. J Renew Sustain Energy 2014;6(2):1-14. doi:10.1063/1.4869568.

[17] Crespo A, Hernandez J, Frandsen S. Survey of modeling methods for wind turbine wakes and wind farms. Wind Energy 2006;2:1-24. doi: 10.1002/(SICI)1099-1824(199901/03)2:1<1::AID-WE16>3.0.CO;2-7.

[18] Kuo JYJ, Romero DA, Beck JC, Amon CH. Wind farm layout optimization on complex terrains - Integrating a CFD wake model with mixed-integer programming. Appl Energy 2016;178:404-14. doi:10.1016/j.apenergy.2016.06.085.

[19] Grassi S, Junghans S, Raubal M. Assessment of the wake effect on the energy production of onshore wind farms using GIS. Appl Energy 2014;136:827-37. doi:10.1016/j.apenergy.2014.05.066.

[20] Veisi AA, Shafiei Mayam MH. Large Eddy Simulation of flow around a single and two in-line horizontal-axis wind turbines. Energy 2017;121:533-44. doi:10.1016/j.energy.2017.01.052.

[21] Adaramola MS, Krogstad PÅ. Experimental investigation of wake effects on wind turbine performance. Renew Energy 2011;36(8):2078-86. doi:10.1016/j.renene.2011.01.024.

[22] Calaf M, Meneveau C, Meyers J. Large eddy simulation study of fully developed wind-turbine array boundary layers. Phys Fluids 2010;22:1-16. doi:10.1063/1.862466.

[23] Li Q, Murata J, Endo M, Maeda T, Kamada Y. Experimental and numerical investigation of the effect of turbulent in flow on a Horizontal Axis Wind Turbine (Part I : Power performance). Energy 2016;113:713-22. doi:10.1016/j.energy.2016.06.138.

[24] Li Q, Kamada Y, Maeda T, Murata J, Nishida Y. Visualization of the flow field and aerodynamic force on a Horizontal Axis Wind Turbine in turbulent inflows. Energy 2016;111:57-67. doi:10.1016/j.energy.2016.05.098.

[25] Xydis G, Koroneos C, Loizidou M. Exergy analysis in a wind speed prognostic model as a wind farm sitting selection tool: a case study in Southern Greece. Appl Energy 2009;86(11):2411-20.

[26] Hu H, Yang Z, Sarkar P. Dynamic wind loads and wake characteristics of a wind turbine model in an atmospheric boundary layer wind. Exp Fluids 2012;52(5):1277-94. doi:10.1007/s00348-011-1253-5. 
[27] Van Dooren MF, Kühn M, Petrovi V, Bottasso CL, Campagnolo F, et al. Demonstration of synchronised scanning lidar measurements of 2D velocity fields in a boundary-layer wind tunnel. Journal of Physics: Conference Series. Vol. 753(7). IOP Publishing, 2016.

[28] Zhong H, Du P, Tang F, Wang L. Lagrangian dynamic large-eddy simulation of wind turbine near wakes combined with an actuator line method. Appl Energy 2015;144:224-33. doi:10.1016/j.apenergy.2015.01.082

[29] Mo JO, Choudhry A, Arjomandi M, Kelso R, Lee YH. Effects of wind speed changes on wake instability of a wind turbine in a virtual wind tunnel using large eddy simulation. J Wind Eng Ind Aerodyn 2013;117:38-56. doi:10.1016/j.jweia.2013.03.007.

[30] Mo JO, Choudhry A, Arjomandi M, Lee YH. Large eddy simulation of the wind turbine wake characteristics in the numerical wind tunnel model. J Wind Eng Ind Aerodyn 2013;112:11-24. doi:10.1016/j.jweia.2012.09.002.

[31] Porté-Agel F, Wu YT, Lu H, Conzemius RJ. Large-eddy simulation of atmospheric boundary layer flow through wind turbines and wind farms. J Wind Eng Ind Aerodyn 2011;99:154-68. doi:10.1016/j.jweia.2011.01.011.

[32] Jimenez A, Crespo A, Migoya E, Garcia J. Advances in large-eddy simulation of a wind turbine wake. J Phys Conf Ser 2007;75:12041. doi:10.1088/1742-6596/75/1/012041.

[33] Liu X, Lu C, Liang S, Godbole A, Chen Y. Vibration-induced aerodynamic loads on large horizontal axis wind turbine blades. Appl Energy 2015;185(2):1109-19. doi:10.1016/j.apenergy.2015.11.080.

[34] Rodrigo JS, et al. IEA-Task 31 WAKEBENCH: Towards a protocol for wind farm flow model evaluation. Part 1: Flowover-terrain models. Journal of Physics: Conference Series. Vol. 524(1). IOP Publishing, 2014.

[35] Moriarty P, et al. IEA-Task 31 WAKEBENCH: Towards a protocol for wind farm flow model evaluation. Part 2: Wind farm wake models. Journal of Physics: Conference Series. Vol. 524(1). IOP Publishing, 2014.

[36] Krogstad PÅ, Eriksen PE. "Blind test" calculations of the performance and wake development for a model wind turbine. Renew Energy 2013;50:325-33. doi:10.1016/j.renene.2012.06.044.

[37] Pierella F, Krogstad PÅ, Sætran L. Blind Test 2 calculations for two in-line model wind turbines where the downstream turbine operates at various rotational speeds. Renew Energy 2014;70:62-77. doi:10.1016/j.renene.2014.03.034.

[38] Tangier JL, Somers DM. NREL airfoil families for HAWTs. Proc ... 1995.॥

[39] Pierella F, Eriksen E, Sætran L. Invitation to the 2012 " Blind test 2 " Workshop Calculations for two wind turbines in line 2012:1-11.

[40] Pope SB. Turbulent Flows 2000:770. doi:10.1179/030801801679395.

[41] Smagorinsky J. General Circulation Experiments With the Primitive Equations. Mon Weather Rev 1963;91:99-164. doi:10.1175/1520-0493(1963)091<0099:GCEWTP>2.3.CO;2.

[42] Alfredsson PH, Dahlberg JA, Vermeulen PEJ. A comparison between predicted and measured data from wind turbine wakes. Wind Eng 1982;6:149-55.

[43] Medici D, Alfredsson PH. Measurements on a wind turbine wake: 3D Effects and bluff body vortex shedding. Wind Energy2006; 9:219-36. doi: 10.1002/we.156.

[44] Kim J. Evolution of a vortical structure associated with the bursting event in a channel flow. Symposium on Turbulent Shear Flows, 1985, University Park, PA, Pennsylvania State University, 1985.

[45] Jeong J, Hussain F. On the identification of a vortex. J. of Fluid Mechanics 2006;285:69-94. doi:10.1017/S0022112095000462.

[46] Perry AE, Chong ME, Cantwell BJ. A general classification of three-dimensional flow fields. J. Physics of Fluids 1990;2:765-77. doi: 10.1063/1.857730.

[47] Hunt JCR, Wray AA, Moin P. Eddies, streams, and convergence zones in turbulent flows. Cent Turbul Res Proc Summer Progr 1988:193-208. doi:CTR-S88.

[48] Maciel Y, Shafiei Mayam MH. Hairpin structures in a turbulent boundary layer under stalled-airfoil-type flow conditions. Progress in Turbulence III 2008;131:199-202. doi:10.1007/978-3-642-02225-8_48. 
[49] Shafiei Mayam MH, Maciel Y. Hairpin structures in a turbulent boundary layer with strong adverse pressure gradient. International Symposium on turbulence and shear flow phenomena 2007.

[50] Shafiei Mayam MH, Maciel Y. Coherent structures in a turbulent boundary layer in stalled-airfoil0type flow conditions. AERO Conference and 54th Annual General Meeting 2007.

[51] Zhou J, Adrian RJ, Balachandar S, Kendall TM. Mechanisms for generating coherent packets of hairpin vortices in channel flow. Journal of Fluid Mechanics 1999:387: 353-96. doi:10.1017/S002211209900467X.

[52] Shafiei Mayam MH. Experimental study of the turbulence structures in a boundary layer subject to a strong adverse pressure gradient. Ph.D. thesis. Laval university, 2009.

[53] Lu H, Porte' -Agel F. Large-eddy simulation of a very large wind farm in a stable atmospheric boundary layer. J. of Phys Fluids 2011;23:65-101.doi:10.1063/1.3589857.

[54] Zahle F, Sørensen NN. Characterization of the unsteady flow in the nacelle region of a modern wind turbine. Wind Energy 2011;14:271-83. doi:10.1002/we.418.

[55] Sherry M, Sheridan J, Jacono DL. Characterisation of a horizontal axis wind turbine's tip and root vortices. Exp Fluids 2013;54. doi:10.1007/s00348-012-1417-y.

[56] Chamorro LP, Porté-Agel F. A wind-tunnel investigation of wind-turbine wakes: Boundary-Layer turbulence effects. Boundary-Layer Meteorol 2009;132:129-49. doi:10.1007/s10546-009-9380-8.

[57] Xie W, Zeng P, Lei L. A novel folding blade of wind turbine rotor for effective power control. Energy Convers Manag 2015;101:52-65. doi:10.1016/j.enconman.2015.05.037. 